\title{
Engine on/off control for dimensioning hybrid electric powertrains via convex optimization
}

\author{
Nikolce Murgovski, Lars Johannesson, and Jonas Sjöberg
}

\begin{abstract}
This paper presents a novel heuristic method for optimal control of mixed-integer problems which for given feasible values of the integer variables are convex in the rest of the variables. The method is based on the Pontryagin's maximum principle and allows the problem to be solved using convex optimization techniques. The advantage of this approach is the short computation time for obtaining a solution near the global optimum, which may otherwise need very long computation time when solved by algorithms guaranteeing global optimum, such as Dynamic Programming.

In this study the method is applied to the problem of battery dimensioning and power split control of a plug-in hybrid electric vehicle where the only integer variable is the engine on/off control, but the method can be extended to problems with more integer variables. The studied vehicle is a city bus which is driven along a perfectly known bus line with a fixed charging infrastructure. The bus can charge either at standstill, or while driving along a tram line (slide-in).

The problem is approached in two different scenarios: first, only the optimal power split control is obtained for several fixed battery sizes; second, both battery size and power split control are optimized simultaneously. Optimizations are performed over four different bus lines and two different battery types, giving solutions that are very close to the global optimum obtained by Dynamic Programming.
\end{abstract}

Index Terms - plug-in/slide-in hybrid electric vehicle, battery sizing, power management, convex optimization, Pontryagin's maximum principle

\section{INTRODUCTION}

Besides the Internal Combustion Engine (ICE), Hybrid Electric Vehicles (HEVs) have an energy buffer, typically a battery and/or a super capacitor, and one or more Electric Machines (EMs). This gives them an additional degree of freedom, compared to conventional vehicles, which allows for a more efficient operation, due to: a possibility to recover braking energy by using the EMs as generators and storing the energy in the buffer; ability to shut down the ICE during idling and low load demands; possibility to run the ICE at more efficient load conditions while storing the excess energy in the buffer. For a detailed overview on hybrid vehicles, see e.g. [1].

Plug-in HEVs (PHEVs) have in addition a charging connector, which allows them to draw electric energy from the grid. The PHEV's that are being considered in public transport are designed to charge from fast-charge docking stations while

The authors are with the Department of Signals and Systems, Chalmers University of Technology, 41296 Gothenburg, Sweden. E-mail: nikolce.murgovski@chalmers.se, larsjo@chalmers.se, jonas.sjoberg@chalmers.se.

L. Johannesson is also with Viktoria Swedish ICT, lars.johannesson@viktoria.se. standing still at stops along the bus line [2], and while driving along sections on the bus line [3], [4]. In [3] the PHEV city bus is inductively charged from underground cables that have been buried along sections of the bus line. In [4] the PHEV, a dual-mode trolley bus, can draw electricity from overhead wires along sections of existing tram lines. Throughout the paper the charge-while-drive sections of the bus line will be called slide-in intervals.

In order to be cost effective, the PHEV city bus is preferred to drive a significant part of the bus line on electric power, even though the charging intervals might be short and the charging infrastructure might be sparsely distributed. This puts hard constraints on the sizing of the energy buffer, i.e. determining power rating and energy capacity, which is not only dependent on the charging infrastructure, but also on the drive patterns, the topography along the bus line, and varying factors, such as fuel and electricity prices. Moreover, a complicating issue when evaluating HEV city buses is that the energy efficiency of the powertrain depends on how well adapted the energy management strategy (power split control) is to the bus line [5]. For PHEV city buses the energy management strategy decides the operating point of the ICE and thereby when and at which rate the energy buffer is to be discharged. When optimizing the PHEV public transportation system based on a dynamic model of the powertrain, a badly designed/adjusted energy management may lead to a non-optimal size of the energy buffer [6]. Hence, to overcome this problem, both the size of the energy buffer and the energy management need to be optimized simultaneously.

The problem of optimal sizing and control of HEVs is traditionally solved by Dynamic Programming (DP) [7], for which vast number of scientific articles are available [8], [9], [10], [11], [12], [13]. The main advantage with DP is the capability to use nonlinear, non-convex models of the components consisting of continuous and integer (mixed-integer) optimization variables. However, a serious limitation of DP is that computation time increases exponentially with the number of state variables [7]. As a consequence, the powertrain model is typically limited to only one or possibly two continuous state variables. Moreover, since DP operates by recursively solving a smaller sub-problem for each time step, the second limitation of DP is that it is not possible to directly include the component sizing into the optimization. Instead, DP must be run in several loops to obtain the optimal control over a grid of component sizes, which further increases computation time.

Another approach, proposed by [14], uses convex optimization for optimal control of HEVs. In this early study 
the powertrain components are expressed with linear models and the optimization problem is a linear program. In a more recent study [15], the strategy is extended to powertrains with quadratic losses for the components, and not only optimal control is obtained, but also simultaneously the energy buffer is sized by solving a semidefinite convex program [16]. The study showed that for a battery with nearly constant voltage within the allowed state of charge interval, the error due to convexifying approximations of the powertrain components is small. However, the disadvantage of this strategy is that it relies on heuristic decision for the integer variables, such as gear, and engine on/off.

This paper is an extension of [15] and proposes a novel heuristic strategy that decides engine on/off control for PHEV powertrains in series topology [1]. The strategy is based on the Pontryagin's maximum principle [17] and requires solving the convex problem iteratively, while using the Hamiltonian [18], [19] to obtain information on the possible improvement in cost from flipping the value of the engine on/off signal at certain time instances. The paper illustrates several examples where the problem of cost optimal battery sizing, investigated for four different bus lines and two different battery types, is solved in less than $17 \mathrm{~min}$ an a personal computer. The results are validated with DP showing less than $0.35 \%$ difference from the global optimum. Moreover, to test the convergence of the algorithm, the problem of optimal control of a powertrain with a fixed battery is solved for 176 different battery sizes. Each optimization needed less than 5 min and achieved a solution within $0.03 \%$ of the global optimum.

The paper is outlined as follows: problem formulation and modeling details are described in Section II; convex modeling and lower bound on the optimization problem are discussed in Section III; the novel heuristic algorithm is depicted in Section IV; examples of optimal battery sizing are given in Section $\mathrm{V}$; and the paper is ended with discussion and conclusion in Section VI and VII, respectively.

\section{PROBLEM FORMULATION}

This section gives background on the bus line and vehicle model, and formulates the PHEV battery sizing and power split control problem.

\section{A. Bus line and vehicle model}

The investigated vehicle is a slide-in/plug-in hybrid electric bus in a series powertrain topology [1], where unlike the conventional vehicles, its combustion engine is completely decoupled from the wheels (Fig. 1). The wheels are propelled by an EM that receives energy from the electric grid, battery, and Engine-Generator Unit (EGU).

The bus is driven on a bus line described by a road gradient and demanded velocity at each point of time (Fig. 2). The bus line model, together with the vehicle inertia, aerodynamic drag and rolling resistance, can be turned into torque $T_{v}(\boldsymbol{n}, t)$ and speed $\omega(t)$ demanded by the EM. The EM, which has torque $\boldsymbol{T}(t)$ (to improve readability decision variables will be marked in bold), is designed to be able to deliver the demanded torque, except during braking when not all torque may be

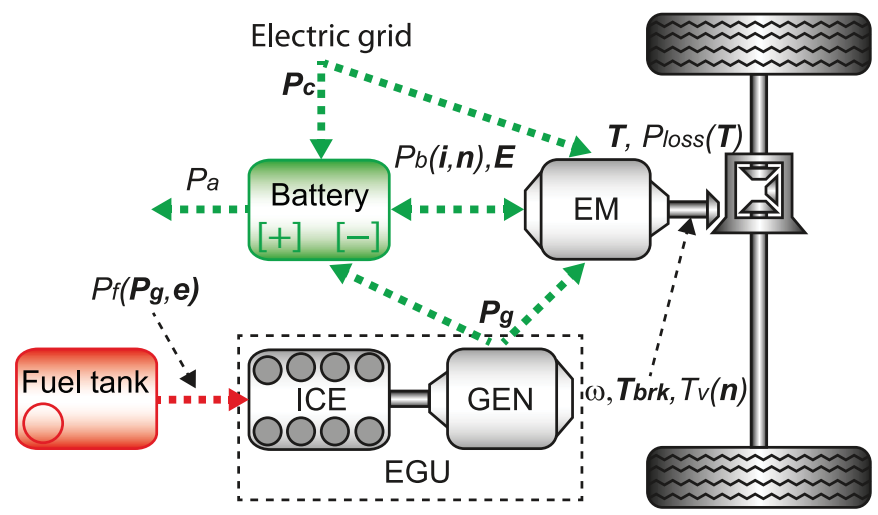

Fig. 1. Series PHEV powertrain model.

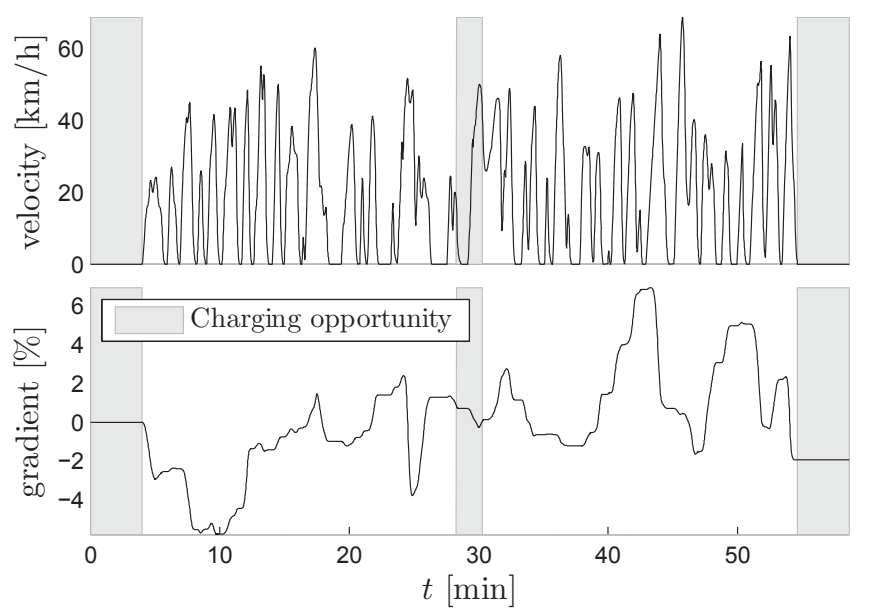

Fig. 2. Bus line model described by demanded velocity and road gradient. The bus line has three charging opportunities, shaded in the figure. The bus can charge 4 min while standing still at each end, and $2 \mathrm{~min}$ while driving along a tram line at about the middle of the bus line.

recuperated to charge the battery, but some portion $\boldsymbol{T}_{\boldsymbol{b r k}}(t) \geq$ 0 is dissipated at the friction brakes, i.e.

$$
\boldsymbol{T}(t)=T_{v}(\boldsymbol{n}, t)-\boldsymbol{T}_{\boldsymbol{b r k}}(t) .
$$

The model considers a demanded torque $T_{v}(\boldsymbol{n}, t)$ that is an affine function on battery mass, hence it is affine on the number of battery cells $\boldsymbol{n}$. A detailed description of $T_{v}(\boldsymbol{n}, t)$ is given in Appendix A.

The powertrain electric path is described by a power balance

$$
\begin{aligned}
& \boldsymbol{T}(t) \omega(t)+P_{\text {loss }}(\boldsymbol{T}(t), t) \\
& \quad=P_{b}(\boldsymbol{i}(t), \boldsymbol{n})+\boldsymbol{P}_{\boldsymbol{g}}(t) \boldsymbol{e}(t)+\boldsymbol{P}_{\boldsymbol{c}}(t) c(t)-P_{a}
\end{aligned}
$$

that relates the EM electric power, left side of the equality, to the battery power $P_{b}(\boldsymbol{i}(t), \boldsymbol{n})$, the EGU power $\boldsymbol{P}_{\boldsymbol{g}}(t)$, the grid charging power $\boldsymbol{P}_{\boldsymbol{c}}(t)$, and the power consumed by auxiliary devices $P_{a}$. The losses of the EM, including losses of the power electronics, are modeled as a quadratic function on $T(t)$

$$
P_{l o s s}(\boldsymbol{T}(t), t)=b_{0}(\omega(t)) \boldsymbol{T}^{\mathbf{2}}(t)+b_{1}(\omega(t)) \boldsymbol{T}(t)+b_{2}(\omega(t))
$$

with speed dependent coefficients where $b_{j}(\omega(t)) \geq 0, j \in$ $\{0,2\}, \forall t \in\left[t_{0}, t_{f}\right]$. The EGU losses are also modeled by a 
quadratic function,

$$
P_{f}\left(\boldsymbol{P}_{\boldsymbol{g}}(t), \boldsymbol{e}(t)\right)=\left(a_{0} \boldsymbol{P}_{\boldsymbol{g}}^{\mathbf{2}}(t)+a_{1} \boldsymbol{P}_{\boldsymbol{g}}(t)+a_{2}\right) \boldsymbol{e}(t)
$$

with $a_{j} \geq 0, j \in\{0,2\}$, where $e(t)$ is a binary signal that is needed to allow zero fuel power, i.e. to remove the idling losses $a_{2}$ when the engine is off. More details on the validity of the EM and EGU models can be found in [15].

The vehicle has three charging opportunities, $4 \mathrm{~min}$ while standing still at each end, and 2 min while sliding in a tram line at about the middle of the bus line. It is assumed, for simplicity, that the chargers have equal maximum power $P_{c \max }$ and a constant charging efficiency $\eta$. The charging opportunities, shaded in Fig. 2, are indicated by a binary signal $c(t)$.

\section{B. Battery model}

The battery consists of $\boldsymbol{n}$ identical cells equally divided in parallel strings, with the strings constructed of cells connected in series. In this study two types of cells are considered, a high energy $44 \mathrm{Ah}$ cell and a high power $10 \mathrm{Ah}$ cell. The cells' open circuit voltage, illustrated in Fig. 3, is approximated by a constant voltage $V$, for simplicity. This gives good fit within the operating state of charge (SOC) range of the batteries, but the method presented in this study can also be applied to batteries with affine voltage-SOC approximation (details on convex modeling can be found in [20]).

Denoting by $\boldsymbol{i}_{\boldsymbol{c}}(t)$ and $R$ the cell current and resistance, respectively, the battery pack power can be expressed as

$$
P_{b}\left(\boldsymbol{i}_{\boldsymbol{c}}(t), \boldsymbol{n}\right)=\left(V \boldsymbol{i}_{\boldsymbol{c}}(t)-R \boldsymbol{i}_{\boldsymbol{c}}^{\mathbf{2}}(t)\right) \boldsymbol{n}
$$

showing that the power of each cell $P_{b}\left(\boldsymbol{i}_{\boldsymbol{c}}(t), \boldsymbol{n}\right) / \boldsymbol{n}$ is equal and does not depend on the configuration of cells (series/parallel). Therefore, the main objective of this paper is to determine the total number of cells in the pack. After the optimal number of cells is obtained, the cells can be configured in parallel strings such that the open circuit voltage of a string fulfills a desired specification. It can be expected that the error due to rounding the total number of cells to a multiple of the number of cells in series will be small if the results point to large number of cells. This will generally be the case if the cells are chosen with very small capacity, as it can be assumed that each cell is constructed by connecting smaller cells in parallel. With this reasoning, the cells can be chosen small enough to consider $\boldsymbol{n}$ as a real-valued variable. (Then, the optimal number of cells can also be interpreted as the optimal pack capacity.)

This paper studies two scenarios with $\boldsymbol{n}$ considered an integer, or a real number. It has been shown in [15] that when $\boldsymbol{n}$ has a real value it is beneficial to replace the cell current in (4) with the pack current $\boldsymbol{i}(t)=\boldsymbol{n} \boldsymbol{i}_{\boldsymbol{c}}(t)$, giving the battery power equation

$$
P_{b}(\boldsymbol{i}(t), \boldsymbol{n})=V \boldsymbol{i}(t)-R \frac{\boldsymbol{i}^{\mathbf{2}}(t)}{\boldsymbol{n}} .
$$

This replaces the non-convex product of two variables $\boldsymbol{i}_{\boldsymbol{c}}(t) \boldsymbol{n}$ in (4) by a quadratic-over-linear function $\boldsymbol{i}^{2}(t) / \boldsymbol{n}$ that is convex in both $\boldsymbol{i}$ and $\boldsymbol{n}$ for $\boldsymbol{n}$ real and strictly positive number.

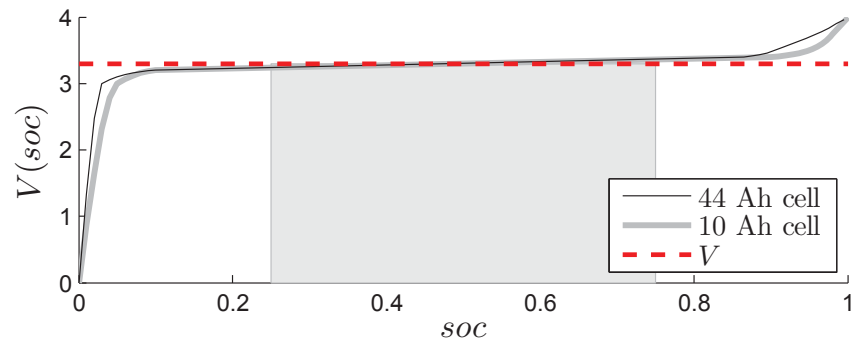

Fig. 3. Model of the battery open circuit voltage. The solid lines represent the original models and the dashed line is the approximation. Good fit is expected in the allowed SOC range represented by the shaded region.

Using the pack current $i(t)$, the battery dynamics can be described by

$$
\dot{\boldsymbol{E}}(t)=-V \boldsymbol{i}(t)
$$

where $\boldsymbol{E}(t)$ is the pack energy.

\section{The mixed-integer optimization problem}

The studied optimization problem is formulated to minimize operational and component costs. The component cost is a linear function on battery cells, $w_{b} \boldsymbol{n}$, while the operational cost refers to cumulative use of fuel and electricity on the bus line, which at each time instant can be represented as

$$
J\left(\boldsymbol{P}_{\boldsymbol{g}}(t), \boldsymbol{P}_{\boldsymbol{c}}(t), \boldsymbol{e}(t)\right)=w_{f} P_{f}\left(\boldsymbol{P}_{\boldsymbol{g}}(t), \boldsymbol{e}(t)\right)+\frac{w_{c}}{\eta} \boldsymbol{P}_{\boldsymbol{c}}(t) \text {. }
$$

The coefficients, $w_{b}$ [currency] and $w_{f}, w_{c}$ [currency/kWh] are used to transform the two costs into a single unit.

The optimization problem can then be summarized as the following nonlinear mixed-integer minimization problem

minimize

$\int_{t_{0}}^{t_{f}} J\left(\boldsymbol{P}_{\boldsymbol{g}}(t), \boldsymbol{P}_{\boldsymbol{c}}(t), \boldsymbol{e}(t)\right) d t+w_{b} \boldsymbol{n}$

subject to, $\forall t \in\left[t_{0}, t_{f}\right]$,

$\boldsymbol{T}(t) \geq \max \left\{T_{\min }(\omega(t)), T_{v}(\boldsymbol{n}, t)\right\}$

$\boldsymbol{T}(t) \omega(t)+P_{\text {loss }}(\boldsymbol{T}(t), t)$ $\leq P_{b}(\boldsymbol{i}(t), \boldsymbol{n})+\boldsymbol{P}_{\boldsymbol{g}}(t) \boldsymbol{e}(t)+\boldsymbol{P}_{\boldsymbol{c}}(t) c(t)-P_{a}$

$\boldsymbol{i}(t) \in\left[i_{\text {cmin }}, i_{\text {cmax }}\right] \boldsymbol{n}$

$\boldsymbol{P}_{\boldsymbol{g}}(t) \in\left[0, P_{\text {gmax }}\right]$

$\boldsymbol{P}_{\boldsymbol{c}}(t) \in\left[0, \eta P_{\text {cmax }}\right]$

$\dot{\boldsymbol{E}}(t)=-V \boldsymbol{i}(t)$

$\boldsymbol{E}(t) \in\left[\right.$ soc $_{\min }$, soc $\left._{\max }\right] Q V \boldsymbol{n}$

$\boldsymbol{E}\left(t_{f}\right)=\boldsymbol{E}\left(t_{0}\right)=\operatorname{soc}_{0} Q V \boldsymbol{n}$

$\boldsymbol{e}(t) \in\{0,1\}$

$\boldsymbol{n}>0$

$\boldsymbol{n} \in \begin{cases}\mathbb{Z}, & \text { in scenario where } \boldsymbol{n} \text { is an integer number } \\ \mathbb{R}, & \text { in scenario where } \boldsymbol{n} \text { is a real number }\end{cases}$

$\boldsymbol{P}_{\boldsymbol{g}}(t), \boldsymbol{P}_{\boldsymbol{c}}(t), \boldsymbol{T}(t), \boldsymbol{E}(t), \boldsymbol{i}(t) \in \mathbb{R}$

where $\boldsymbol{P}_{\boldsymbol{g}}(t), \boldsymbol{P}_{\boldsymbol{c}}(t), \boldsymbol{T}(t), \boldsymbol{E}(t), \boldsymbol{e}(t), \boldsymbol{i}(t)$ and $\boldsymbol{n}$ are optimization variables. In the view of optimal control, that will 
be further discussed in Section IV, $\boldsymbol{P}_{\boldsymbol{g}}(t), \boldsymbol{P}_{\boldsymbol{c}}(t), \boldsymbol{T}(t), \boldsymbol{e}(t)$ and $\boldsymbol{i}(t)$ are control signals, $\boldsymbol{E}(t)$ is a state, and $\boldsymbol{n}$ is a design parameter. The optimization includes bounds on the cell current $i_{c \min }, i_{c \max }$, maximum EGU power $P_{\text {gmax }}$, initial SOC $s o c_{0}$, allowed SOC range $s o c_{\min }, s o c_{\max }$, and a speed dependent bound on the EM generating torque $T_{\min }(\omega(t))$. The battery cell capacity is denoted by $Q$ and the optimization requires charge sustaining operation by (7i). The initial and final time of the bus line are denoted by $t_{0}$ and $t_{f}$, respectively.

It can be noticed that (1) and (2) have been relaxed with inequalities in (7b) and (7c) and the braking torque has been taken outside the optimization problem. The reason for doing this will become clear in the following section, but at the moment we claim that although the relaxation does change the optimization problem, it does not change the optimal result. Namely, in (7c) the battery and the generator are allowed to produce more power than the electrical power needed by the EM to drive the bus. Similarly (7b) allows the EM to generate more mechanical power than needed. It is obvious that at the optimum these constraints will hold with equality, since otherwise energy will be wasted unnecessarily. The only exception is during braking when ( $7 b)$ can be satisfied with inequality, only if the optimal battery is small and the brake recuperation power is limited by the battery through (7d), rather than by the EM. In either case of bounded recuperation power, the friction brakes will be used to compensate for the remaining braking power. Hence, after the optimization is finished, the optimal braking power can be derived directly from (1) and (2).

The battery dimensioning problem in this paper is approached in two different scenarios. In the first scenario the variable $\boldsymbol{n}$ is taken outside the optimization, such that the mixed-integer optimal control problem (7) is solved over a grid of fixed battery sizes. The disadvantage of this scenario is the long computation time due to the iterative solution of the optimal control problem.

In the second scenario $\boldsymbol{n}$ is considered a real number and in (7) the battery is dimensioned simultaneously when obtaining the optimal control. An advantage of this approach is that it might give shorter computation time, because a loop over battery sizes is not needed.

\section{CONVEX OPTIMIZATION}

This section gives a brief background on convex optimization and discusses how convex optimization can be used to find a lower bound to the solution of the mixed-integer optimization problem.

\section{A. Definition for a convex problem}

A convex problem, in its general form, can be written as

$$
\begin{array}{cl}
\operatorname{minimize} & f_{0}(x) \\
\text { subject to } & f_{i}(x) \leq 0, \quad i=1, \ldots, m \\
& h_{j}(x)=0, \quad j=1, \ldots, p \\
& x \in \mathcal{X}
\end{array}
$$

where $\mathcal{X} \subseteq \mathbb{R}^{n}$ is a convex set, $f_{i}(x)$ are convex functions and $h_{j}(x)$ are affine in the vector of decision variables $\boldsymbol{x}$
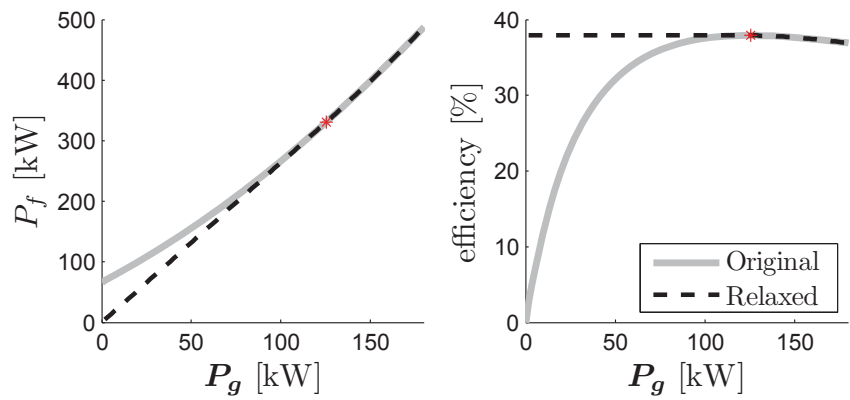

Fig. 4. The original and relaxed EGU models. For generator power lower than the power at peak efficiency, the efficiency of the relaxed model is equal to the peak efficiency of the original model. Above this point the efficiency of the relaxed model follows the efficiency of the original model.

[16]. For the dimensioning problem (7), the vector $x$ will be very large (thousands of elements), because it will include the optimization variables in (7) for all the time instances $t_{k} \in \mathcal{T}$ of a typically large discrete set $\mathcal{T}$. Moreover, the problem (7) has only affine functions in equality constraints and nonlinear functions in inequality constraints (hence the reason for relaxing (1) and (2)), but it is not convex. This is because even when $\boldsymbol{n}$ is considered a real number, the control variable $\boldsymbol{e}(t)$ belongs to an integer set. Besides, the product $\boldsymbol{P}_{\boldsymbol{g}}(t) \boldsymbol{e}(t)$ is not a convex function, even if $\boldsymbol{e}(t)$ could be relaxed to a real number.

\section{B. Lower bound to the mixed-integer problem}

A common approach for optimizing mixed-integer problems is to relax the integer variables to real value variables [21]. The obtained solution of the relaxed problem is then a lower bound to the mixed-integer problem. Moreover, if the optimal values of the relaxed variables are nearly integer, then techniques exist, not too computationally demanding, to obtain the optimal solution of the mixed-integer problem [21]. This section investigates whether or not the relaxation of the engine on/off control should be used for obtaining the optimal solution of the mixed-integer, dimensioning and control problem (7), when $\boldsymbol{n}$ is considered a real number.

With $\boldsymbol{e}(t)$ relaxed to a real value, a convex form of (7) can be obtained by introducing a variable change $\tilde{\boldsymbol{P}}_{\boldsymbol{g}}(t)=$ $\boldsymbol{P}_{\boldsymbol{g}}(t) \boldsymbol{e}(t)$. This will replace (3), (7c), (7e) and (7j) with

$$
\begin{aligned}
& P_{f}\left(\tilde{\boldsymbol{P}}_{\boldsymbol{g}}(t), \boldsymbol{e}(t)\right)=a_{0} \frac{\tilde{\boldsymbol{P}}_{\boldsymbol{g}}^{\mathbf{2}}(t)}{\boldsymbol{e}(t)}+a_{1} \tilde{\boldsymbol{P}}_{\boldsymbol{g}}(t)+a_{2} \boldsymbol{e}(t) \\
& \boldsymbol{T}(t) \omega(t)+P_{\text {loss }}(\boldsymbol{T}(t), t) \\
& \leq P_{b}(\boldsymbol{i}(t), \boldsymbol{n})+\tilde{\boldsymbol{P}}_{\boldsymbol{g}}(t)+\boldsymbol{P}_{\boldsymbol{c}}(t) c(t)-P_{a} \\
& \tilde{\boldsymbol{P}}_{\boldsymbol{g}}(t) \in\left[0, \boldsymbol{e}(t) P_{g, \max }\right] \\
& \boldsymbol{e}(t) \in(0,1]
\end{aligned}
$$

where $e(t)$ is limited to strictly positive to avoid division by zero in $(8 \mathrm{a})$.

This convex problem can be easily solved with publicly available tools, e.g. SeDuMi [22], but the implications from the relaxation of $\boldsymbol{e}(t)$ can also be reasoned analytically. It can be easily found that the optimal value $e^{*}(t)$ that minimizes the 
fuel power (8a) can be expressed as a function of the generator power

$$
\boldsymbol{e}^{*}(t)=\min \left\{\tilde{\boldsymbol{P}}_{\boldsymbol{g}}(t) \sqrt{a_{0} / a_{2}}, 1\right\} .
$$

When this is replaced back to (8a), an expression can be obtained

$P_{f}^{*}\left(\tilde{\boldsymbol{P}}_{\boldsymbol{g}}(t)\right)= \begin{cases}\tilde{\boldsymbol{P}}_{\boldsymbol{g}}(t)\left(2 \sqrt{a_{0} a_{2}}+a_{1}\right), & \tilde{\boldsymbol{P}}_{\boldsymbol{g}}(t) \leq \sqrt{a_{2} / a_{0}} \\ a_{0} \tilde{\boldsymbol{P}}_{\boldsymbol{g}}^{\mathbf{2}}(t)+a_{1} \tilde{\boldsymbol{P}}_{\boldsymbol{g}}(t)+a_{2}, & \text { otherwise. }\end{cases}$

which indicates that the relaxation of $\boldsymbol{e}(t)$ causes significant changes to the original EGU model, as illustrated in Fig. 4. Namely, the relaxed EGU model removes an important limitation of the ICE, the low efficiency during idling. Without this limitation, the optimal control $e^{*}(t)$ is not mainly binary, but a continuous value in $(0,1]$ that smoothly follows the changes in demanded power. Hence, besides using it as a lower bound, the solution of this relaxed problem will not be used for obtaining the solution of the mixed-integer problem.

\section{Heuristics BASED ON COSTATE}

This section introduces a novel strategy for optimal control of the studied mixed-integer problem. The strategy starts by deciding feasible values for the integer variable $e(t)$ at each point of time. Then, the optimization problem (7) becomes a convex sub-problem that can be solved to obtain the optimal values of the rest of the optimization variables. The optimal solution of the sub-problem is used to iteratively improve the initial choice of $e(t)$ in direction of minimizing the optimization cost of the convex sub-problem solved in a loop. A method to obtain an initial feasible solution for $\boldsymbol{e}(t)$ is discussed later in Section IV-B.

At the optimal solution of the sub-problem, the Hamiltonian [18], [19] is investigated

$$
\mathcal{H}^{*}(\cdot)=J\left(\boldsymbol{P}_{\boldsymbol{g}}^{*}(t), \boldsymbol{P}_{\boldsymbol{c}}^{*}(t), \boldsymbol{e}(t)\right)-V \boldsymbol{\lambda}^{*}(t) \boldsymbol{i}^{*}(t)
$$

that gives an equivalent fuel-electricity cost at each time instance on the bus line. The symbol $\cdot$ in $\mathcal{H}^{*}(\cdot)$ represents a compact notation of a function of decision variables, and $\boldsymbol{\lambda}(t)$ is the costate of the system, also known as adjoint state, or Lagrange multiplier [23], [24]. In the optimal control of hybrid vehicles, $-\boldsymbol{\lambda}(t)$ is also referred to as equivalence factor [25], [26], since it translates the electric energy used by the EM to an equivalent fuel consumption. In this study the unit of $\boldsymbol{\lambda}(t)$ is [currency/kWh].

\section{A. The costate heuristic method}

The idea of the costate method is built upon the assumption that the optimal costate of the convex sub-problem is close to the globally optimal costate of the mixed-integer problem. First an initial feasible value for $e(t)$ is decided, the convex sub-problem is solved and the costate $\lambda^{*}(t)$ is obtained. Then this costate is used to modify the initial integer control $e(t)$, which when used again in the convex sub-problem may further decrease the cost.

In order to decide at which time instances $\boldsymbol{e}(t)$ is to be modified, a so called Complementary Hamiltonian is constructed
$\tilde{\mathcal{H}}(\neg \boldsymbol{e}(t), \cdot)$, where the engine on/off signal has flipped value at each time instance (here $e(t)$ is also used as a Boolean variable). In this context $\tilde{\mathcal{H}}(\neg \boldsymbol{e}(t), \cdot)$ is a measure of the possible decrease in cost by changing the integer control $\boldsymbol{e}(t)$ at certain time instances. At a predefined number, $N_{f}$, of time instances with highest difference $\mathcal{H}^{*}(e(t), \cdot)-\tilde{\mathcal{H}}^{*}(\neg e(t), \cdot)$, the value of $e(t)$ is flipped and the convex sub-problem is solved again for the recently obtained $e(t)$. This procedure is repeated while there are improvements in the cost and it can be summarized as follows:

1) A feasible solution for the integer control $e(t)$ is decided and the globally minimal cost is assigned infinity, such that the first feasible solution will be accepted.

2) For the choice of $e(t)$ the optimal solution of the convex sub-problem is obtained. If the problem is infeasible or there is no improvement in cost, then go to 5.

3) From the solution of the sub-problem, the optimal costate $\lambda^{*}(t)$ is obtained. Using the costate, $\mathcal{H}^{*}(\boldsymbol{e}(t), \cdot)$ is computed and $\tilde{\mathcal{H}}(\neg e(t), \cdot)$ is minimized.

4) The value of $e(t)$ is flipped at $N_{f}$ time instances with the highest difference $\mathcal{H}^{*}(\boldsymbol{e}(t), \cdot)-\tilde{\mathcal{H}}^{*}(\neg \boldsymbol{e}(t), \cdot)$, and the algorithm goes back to step 2 .

5) If $N_{f}>1$, then $N_{f}=\operatorname{round}\left(N_{f} / 2\right)$, the last change of $\boldsymbol{e}(t)$ is canceled, and the algorithm goes back to 4 . Exit otherwise.

The initial value for $N_{f}=N_{\text {init }}$ is an engineering decision that may affect the number of iterations needed by the algorithm. If the initial solution is known to be close to the global optimum, then $N_{\text {init }}$ can be chosen rather small. If the initial solution is known to be far from the global optimum, or if there is no knowledge about it, then $N_{\text {init }}$ can be as large as $50 \%$ of the number of time samples in the bus line. However, choosing a large value for $N_{\text {init }}$ will not significantly degrade the performance of the algorithm. In a worst case scenario where $N_{\text {init }}$ is large and the initial engine on/off control differs from the global optimum in only one time instance, the algorithm will need to solve the convex sub-problem $\log _{2} N_{\text {init }}$ times before $N_{f}$ decreases to 1 and a change in $\boldsymbol{e}(t)$ is eventually performed.

Later, in the examples in Section $\mathrm{V}$, the initial value for $N_{f}$ has been chosen half the number of times samples in the bus line.

\section{B. A feasible engine on/off control}

If the mixed-integer problem (7) has a feasible solution, then a trivial solution is choosing $e(t)$ that never turns off the engine. A better solution is to turn on the engine only for high power demands where the EGU is more efficient. Such a solution has been proposed in [15], where the engine is turned on when the power $P_{\text {base }}(t)$ of the vehicle without the weight of the battery exceeds a certain threshold $P_{o n}^{*}$.

Assuming that within the slide-in interval the vehicle will be mainly driven by grid power, a feasible on/off control is

$$
e(t)= \begin{cases}1, & P_{\text {base }}(t)-\eta P_{\text {cmax }} c(t)>P_{o n}^{*} \\ 0, & \text { otherwise }\end{cases}
$$




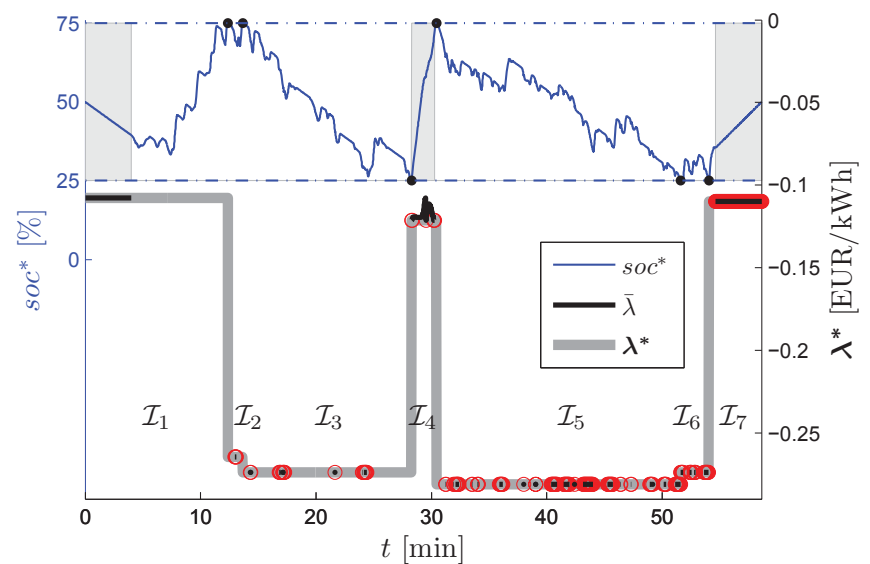

Fig. 5. Battery SOC on the left axis and costate on the right axis. The costate is a piecewise constant function where the different pieces belong to the time intervals $\mathcal{I}_{k}, k=1, \ldots, 7$. The time instances where the value of the costate can be obtained from (14) are represented by circles. Charging opportunities are depicted by shaded regions.

The optimal power threshold $P_{o n}^{*}$ is different for different bus lines. For each bus line $P_{\text {on }}^{*}$ can be found as the threshold that gives a feasible solution and minimizes the cost of the convex sub-problem iteratively solved for several gridded (discrete) values $P_{o n_{j}} \in\left[0, P_{\text {gmax }}\right]$ within the power range of the vehicle.

Note that with this procedure the optimal battery size and powertrain control can be obtained simultaneously. This is because for a given $\boldsymbol{e}(t)$ the optimization sub-problem (7) is convex in $\boldsymbol{n}$ as well as in the optimization variables $\boldsymbol{P}_{\boldsymbol{g}}(t), \boldsymbol{P}_{\boldsymbol{c}}(t), \boldsymbol{T}(t), \boldsymbol{E}(t)$ and $\boldsymbol{i}(t)$. Moreover, it has been shown in [15] that this solution is close to the global optimum, both in total optimization cost and battery size. This is also observed in the results in Section V-D.

\section{Computing the costate}

Most solvers of convex problem, e.g. SeDuMi [22], provide the Lagrangian dual variables together with the primal optimal solution. Then, the costate required in (9) will be the dual variable associated to constraint $(7 \mathrm{~g})$. In this section, however, the costate will be derived using the Pontryagin's maximum principle [17]. This gives a better insight on the nature of the costate function and on the time instances at which the costate is not strictly defined. For didactic reasons, the process is described through a snapshot, see Fig. 5, taken during the iterations of the costate algorithm for an example of optimal battery dimensioning and control of a PHEV bus. The snapshot depicts the optimal SOC and costate trajectory of the convex sub-problem of the bus driven on the bus line shown in Fig. 2 , with a battery consisting of 10 Ah cells.

1) Necessary conditions: The necessary condition for an extremum of the Hamiltonian

$$
\frac{d \boldsymbol{\lambda}^{*}(t)}{d t}=-\left(\frac{\partial \mathcal{H}(\cdot)}{\partial \boldsymbol{E}(t)}\right)^{*}=0, \quad \forall t \in \mathcal{T}_{E}
$$

reveals that the costate is constant in time intervals $\mathcal{T}_{E}$ without active state constraints, since $\mathcal{H}(\cdot)$ does not depend explicitly on $\boldsymbol{E}(t)$. By introducing an operator free $(\boldsymbol{E}(t))$ that gives "true" if ( $7 \mathrm{~h})$ is not active, the set $\mathcal{T}_{E}$ can be mathematically described by

$$
\mathcal{T}_{E}=\left\{t \in\left[t_{0}, t_{f}\right] \mid \operatorname{free}(\boldsymbol{E}(t))\right\} .
$$

The value of the costate in these intervals can be obtained from the second necessary condition for an extremum

$$
\left(\frac{\partial \mathcal{H}(\cdot)}{\partial \boldsymbol{u}(t)}\right)^{*}=0, \quad \forall t \in\left\{\mathcal{T}_{1} \cup \mathcal{T}_{2}\right\}
$$

with $\boldsymbol{u}(t)=\left[\begin{array}{llll}\boldsymbol{i}(t) & \boldsymbol{P}_{\boldsymbol{c}}(t) & \boldsymbol{P}_{\boldsymbol{g}}(t) & \boldsymbol{T}(t)\end{array}\right]^{T}$, at time instances $t \in\left\{\mathcal{T}_{1} \cup \mathcal{T}_{2}\right\}$ without active constraints on control signals of $\boldsymbol{u}^{*}(t)$. Moreover, the convexity of the problem ensures that $\mathcal{H}^{*}(\cdot)$ is in fact a minumum, i.e.

$$
\frac{\partial^{2} \mathcal{H}(\cdot)}{\partial \boldsymbol{u}^{2}(t)} \geq 0, \quad \forall t \in\left[t_{0}, t_{f}\right] .
$$

For a chosen on/off control $e(t)$ the convex optimization will find $\boldsymbol{u}^{*}(t)$ that minimizes the total cost of the sub-problem and the only unknown in (13) will be $\lambda^{*}(t)$. It can be shown (detailed in Appendix B) that the optimal costate, illustrated by circles in Fig. 5, is

$\boldsymbol{\lambda}^{*}(t)= \begin{cases}-w_{f}\left(2 a_{o} \boldsymbol{P}_{\boldsymbol{g}}^{*}(t)+a_{1}\right)\left(1-\frac{2 R}{\boldsymbol{n}^{*} V} \boldsymbol{i}^{*}(t)\right), & t \in \mathcal{T}_{1} \\ -\frac{w_{c}}{\eta}\left(1-\frac{2 R}{\boldsymbol{n}^{*} V} \boldsymbol{i}^{*}(t)\right), & t \in \mathcal{T}_{2}\end{cases}$

with the sets $\mathcal{T}_{1}, \mathcal{T}_{2}$ described by

$$
\begin{aligned}
\mathcal{T}_{1}= & \left\{t \in\left[t_{0}, t_{f}\right] \mid \boldsymbol{e}(t) \wedge \neg c(t) \wedge \operatorname{free}\left(\boldsymbol{i}^{*}(t)\right) \wedge \operatorname{free}\left(\boldsymbol{P}_{\boldsymbol{g}}^{*}(t)\right)\right\} \\
\mathcal{T}_{2}= & \left\{t \in\left[t_{0}, t_{f}\right] \mid \operatorname{free}\left(\boldsymbol{i}^{*}(t)\right) \wedge \operatorname{free}\left(\boldsymbol{P}_{\boldsymbol{c}}^{*}(t)\right) \wedge c(t)\right. \\
& \left.\wedge\left(\boldsymbol{e}(t) \wedge \operatorname{free}\left(\boldsymbol{P}_{\boldsymbol{g}}^{*}(t)\right) \vee \neg \boldsymbol{e}(t)\right)\right\}
\end{aligned}
$$

where free $(\boldsymbol{i}(t))$, free $\left(\boldsymbol{P}_{\boldsymbol{g}}(t)\right)$ and free $\left(\boldsymbol{P}_{\boldsymbol{c}}(t)\right)$ give true if the constraints (7d), (7e) and (7f), respectively, are not active.

Because $\lambda^{*}(t)$ is a piecewise constant function, it can be completely reconstructed along the bus line if each of the intervals $\mathcal{I}_{k}$ contains at least one time instance $t \in\left\{\mathcal{T}_{1} \cup \mathcal{T}_{2}\right\}$.

Note that (13) may hold with equality at other time instances not belonging to $\mathcal{T}_{1}$ and $\mathcal{T}_{2}$, when $\boldsymbol{u}^{*}(t)$ is inside the bounded region and the optimal solution is singular. However, the singularity of the solution will not be explicitly studied. Instead, to obtain more information on the costate, its upper bound will be investigated.

2) Upper bound to the costate: When no time instance in $\mathcal{I}_{k}$ belongs to $\mathcal{T}_{1}$ or $\mathcal{T}_{2}$, the value of the costate in $\mathcal{I}_{k}$ cannot be obtained from (14). Such a situation is depicted in $\mathcal{I}_{1}$ in Fig. 5 , where it was found optimal to not charge from the grid during the first charging opportunity. (With the high power battery cells the vehicle can recuperate the free of charge braking energy available at the beginning of the cycle due to the negative road gradient.)

In these cases the costate is assigned an upper bound $\bar{\lambda}(t)$

$$
\begin{aligned}
& \lambda^{*}(t)=\min \mathcal{L}_{k}, \quad \forall t \in \mathcal{I}_{k} \\
& \mathcal{L}_{k}=\left\{\bar{\lambda}(t) \mid t \in \mathcal{I}_{k}\right\}
\end{aligned}
$$


with $\bar{\lambda}(t)$ computed exactly as in (14), but with $\mathcal{T}_{1}, \mathcal{T}_{2}$ replaced by

$$
\begin{aligned}
& \mathcal{T}_{3}=\left\{t \in\left[t_{0}, t_{f}\right] \mid \boldsymbol{e}(t) \wedge \neg c(t)\right\} \\
& \mathcal{T}_{4}=\left\{t \in\left[t_{0}, t_{f}\right] \mid c(t)\right\}
\end{aligned}
$$

respectively. The upper bound $\overline{\boldsymbol{\lambda}}(t)$ can be interpreted as the value of the costate that will minimize the Hamiltonian in $\mathcal{I}_{k}$ with a control $\boldsymbol{u}^{*}(t)$ that is free, but very close to the bounds (if $\overline{\boldsymbol{\lambda}}(t)$ is indeed the optimal costate in some time interval, i.e. $\overline{\boldsymbol{\lambda}}(t)=\boldsymbol{\lambda}^{*}(t)$, then the interval is a singular arc [27]).

Note that although $\lambda^{*}(t)$ is constant in $\mathcal{I}_{k}, \bar{\lambda}(t)$ may not be constant, see e.g. $\mathcal{I}_{4}$ in Fig. 5.

3) Intervals with undefined costate: In time intervals $\mathcal{I}_{k}$ where no time instance belongs to $\bigcup_{j=1}^{4} \mathcal{T}_{j}$, neither the costate, nor its upper bound can be computed. Experiments showed that such intervals are rare. Nevertheless, these intervals have been given highest priority in the list of time instances where $\boldsymbol{e}(t)$ will be flipped. This action can be seen as a deliberate disturbance of $\boldsymbol{e}(t)$ that may give more constructive information on the next iteration of the costate heuristic algorithm, i.e. in the next iteration some of these time instance may belong to $\bigcup_{j=1}^{4} \mathcal{T}_{j}$.

The set of all time instances where the costate is undefined is denoted by $\mathcal{T}_{u}$.

\section{The Complementary Hamiltonian}

The Complementary Hamiltonian $\tilde{\mathcal{H}}(\neg e(t), \cdot)$, involved in step 4) of the costate algorithm, is used to give an indication of what can be gained by flipping the value of the integer control $\boldsymbol{e}(t)$. When minimizing $\tilde{\mathcal{H}}(\neg e(t), \cdot)$, the electric energy equivalence to fuel consumption, i.e. the costate $\lambda^{*}(t)$, is considered to be equal to the one that minimizes the Hamiltonian $\mathcal{H}(\boldsymbol{e}(t), \cdot)$. Moreover, for the problem of simultaneous dimensioning and control, it is also assumed that the battery size $\boldsymbol{n}^{*}$ will stay equal to the one used in $\mathcal{H}^{*}(\boldsymbol{e}(t), \cdot)$ (this is further discussed in Section IV-E). Thus, the optimization problem that minimizes the Complementary Hamiltonian can be formulated as a convex problem

$$
\begin{aligned}
& \text { minimize } \\
& \int_{\mathcal{T}_{5}}\left(J\left(\boldsymbol{P}_{\boldsymbol{g}}(t), \boldsymbol{P}_{\boldsymbol{c}}(t), \neg \boldsymbol{e}(t)\right)-\boldsymbol{\lambda}^{*}(t) V \boldsymbol{i}(t)\right) d t \\
& \text { subject to (7b)-(7f), } \forall t \in \mathcal{T}_{5}
\end{aligned}
$$

with optimization variables $\boldsymbol{P}_{\boldsymbol{g}}(t), \boldsymbol{P}_{\boldsymbol{c}}(t)$ and $\boldsymbol{i}(t)$. The set $\mathcal{T}_{5}$ includes the time instances where $\boldsymbol{\lambda}^{*}(t)$ is obtained and where the battery and EGU can meet the power demand, i.e.

$$
\begin{aligned}
\mathcal{T}_{5}= & \left\{t \in\left[t_{0}, t_{f}\right] \backslash \mathcal{T}_{u} \mid T_{v}\left(\boldsymbol{n}^{*}, t\right) \omega(t)\right. \\
& +P_{a}+P_{\text {loss }}\left(T_{v}\left(\boldsymbol{n}^{*}, t\right), t\right) \\
& \left.\leq P_{\text {bmax }}+P_{\text {gmax }} \neg \boldsymbol{e}(t)+\eta P_{\text {cmax }} c(t)\right\}
\end{aligned}
$$

with the maximum battery power found as

$$
P_{b \max }=\boldsymbol{n}^{*} \min \left\{V i_{\text {cmax }}-R i_{c \max }^{2}, \frac{V^{2}}{4 R}\right\} .
$$

At time instances $t \notin \mathcal{T}_{5}, \tilde{\mathcal{H}}^{*}(\cdot)$ has been assigned an infinite cost making these time instances the least desirable choices for flipping the engine on/off control, as they will lead to infeasible solution.

\section{E. Mixed-integer control problems with design parameters}

When minimizing the Complementary Hamiltonian in (15) the battery size $\boldsymbol{n}^{*}$ is kept equal to the one used in $\mathcal{H}^{*}(\boldsymbol{e}(t), \cdot)$. The reason for doing this is to obtain a control problem without design parameters that could be easily analyzed using the classical Optimal Control Theory [27]. A more detailed investigation on how to include the design parameter $\boldsymbol{n}$ when obtaining the extremum of $\tilde{\mathcal{H}}(\cdot)$ will be carried on in future studies. This will be especially relevant if additional powertrain components, such as EM, EGU, ICE, are to be dimensioned simultaneously.

As a consequence, it can be expected that the Complementary Hamiltonian may give indications on how to improve the initial integer control, but it may not give indications on how to improve the initial battery size in two consecutive iterations of the proposed algorithm. For this reason, the initial battery size of the simultaneous dimensioning and control sub-problem (7) should be chosen close to the globally optimal battery size. A strategy for obtaining such an initial solution for the studied problem has been given in Section IV-B, and is further verified in the example in Section V-D. However, for a general parameter design and control problem, it may not be easy to find such a solution. In that case, the proposed costate method could be used by solving the control problem iteratively for several fixed values of the design parameters.

\section{EXAMPLES OF OPTIMAL CONTROL AND BATTERY DIMENSIONING}

This section gives several examples of optimal battery dimensioning and control of a PHEV bus. To obtain solutions (and lower bounds) to these examples, the following optimization methods/setups have been implemented.

- Dynamic Programming (DP) is used for obtaining the global optimum of the mixed integer problem (7) for several fixed battery sizes. The solution from DP is used only for validation purposes. Details concerning its implementation are given in Appendix C.

- The proposed costate heuristic method is used for obtaining the optimal mixed-integer control for battery sizes that were also used in DP. In each step of the algorithm, the Hamiltonian is minimized solving the convex optimal control sub-problem (7) with both $\boldsymbol{e}(t)$ and $\boldsymbol{n}$ having fixed values. In the rest of the paper this convex subproblem will be referred to as CF (Convex, Fixed battery).

- The proposed costate heuristic method is also used for solving the problem of simultaneous dimensioning and control. In each iteration of the algorithm the convex subproblem (7) is solved where $\boldsymbol{e}(t)$ is fixed and $\boldsymbol{n}$ is a realvalued optimization variable. This convex sub-problem will be referred to as CS (Convex, Sizing battery).

- The above two instances of the costate heuristic method include minimization of the Complementary Hamiltonian. 
TABLE I

RESUlTS FROM OPTIMIZATION WITH DYNAMIC PROGRAMMING AND CONVEX OPTIMIZATION.

\begin{tabular}{|c|c|c|c|c|c|c|c|c|c|c|}
\hline Battery cell capacity & & & & $\mathrm{Ah}$ & & & & & & \\
\hline Bus line & & L1 & L2 & L3 & L4 & L1 & L2 & L3 & L4 & \\
\hline & $n$ & 157 & 92 & 237 & 296 & 138 & 121 & 174 & 168 & \\
\hline & $J_{\text {total }}$ & 45.75 & 39.22 & 46.37 & 52.78 & 30.85 & 23.60 & 24.42 & 31.04 & EUR/100km \\
\hline Dynamic Programming (DP) & $J_{f u e l}$ & 29.85 & 27.24 & 20.24 & 22.46 & 20.72 & 11.53 & 8.39 & 16.57 & EUR/100km \\
\hline for fixed battery sizes. & $J_{e l}$ & 4.04 & 5.03 & 8.22 & 7.95 & 2.79 & 5.62 & 6.78 & 5.53 & EUR/100km \\
\hline & $J_{b a t}$ & 11.86 & 6.95 & 17.91 & 22.36 & 7.34 & 6.44 & 9.26 & 8.94 & EUR/100km \\
\hline & $c_{\text {rate }}$ & 36.48 & 24.53 & 49.14 & 62.14 & 9.41 & 21.53 & 29.58 & 29.52 & $\%$ \\
\hline & $n$ & 157 & 92 & 237 & 296 & 138 & 121 & 174 & 168 & \\
\hline Costate heuristic method for & $J_{\text {total }}$ & 45.75 & 39.21 & 46.37 & 52.76 & 30.85 & 23.60 & 24.42 & 31.03 & EUR/100km \\
\hline fixed battery sizes. The & $J_{\text {fuel }}$ & 29.84 & 27.22 & 20.24 & 22.43 & 20.72 & 11.53 & 8.38 & 16.57 & EUR/100km \\
\hline convex sub-problem used in & $J_{e l}$ & 4.05 & 5.04 & 8.22 & 7.97 & 2.79 & 5.62 & 6.78 & 5.53 & EUR/100km \\
\hline the method is CF. & $J_{b a t}$ & 11.86 & 6.95 & 17.91 & 22.36 & 7.34 & 6.44 & 9.26 & 8.94 & EUR/100km \\
\hline & $\delta$ & 0.01 & -0.02 & -0.00 & -0.03 & 0.01 & -0.00 & -0.03 & -0.01 & $\%$ \\
\hline & $n$ & 156.93 & 78.11 & 254.96 & 304.97 & 137.93 & 121.53 & 174.90 & 168.13 & \\
\hline Costate heuristic method for & $J_{\text {total }}$ & 45.76 & 39.30 & 46.52 & 52.84 & 30.84 & 23.60 & 24.42 & 31.03 & EUR/100km \\
\hline $\begin{array}{l}\text { Simultaneous dimensionis } \\
\text { and control. The convex }\end{array}$ & $J_{\text {fuel }}$ & 29.86 & 28.71 & 18.81 & 21.63 & 20.72 & 11.49 & 8.31 & 16.56 & EUR/100km \\
\hline sub-problem used in the & $J_{e l}$ & 4.05 & 4.69 & 8.45 & 8.17 & 2.79 & 5.64 & 6.80 & 5.53 & EUR/100km \\
\hline method is CS. & $J_{b a t}$ & 11.86 & 5.90 & 19.26 & 23.04 & 7.34 & 6.47 & 9.31 & 8.95 & EUR/100km \\
\hline & $\delta$ & 0.02 & 0.20 & 0.32 & 0.12 & -0.02 & -0.00 & -0.03 & -0.01 & $\%$ \\
\hline & $n$ & 158.14 & 78.43 & 254.96 & 313.25 & 137.93 & 121.53 & 184.73 & 168.13 & \\
\hline Initial solution & $J_{\text {total }}$ & 45.77 & 39.36 & 46.54 & 53.08 & 31.11 & 23.61 & 24.46 & 31.04 & EUR/100km \\
\hline costate method using CS. & $J_{\text {fuel }}$ & 29.75 & 28.74 & 18.82 & 21.06 & 20.98 & 11.51 & 7.65 & 16.57 & EUR/100km \\
\hline The initial engine on/off & $J_{e l}$ & 4.07 & 4.70 & 8.45 & 8.35 & 2.79 & 5.64 & 6.98 & 5.53 & EUR/100km \\
\hline control is found by (10). & $J_{b a t}$ & 11.95 & 5.93 & 19.26 & 23.67 & 7.34 & 6.47 & 9.83 & 8.95 & EUR/100km \\
\hline & $\delta$ & 0.05 & 0.37 & 0.37 & 0.57 & 0.85 & 0.07 & 0.15 & 0.02 & $\%$ \\
\hline Lower bound t & $n$ & 66.28 & 4.52 & 108.20 & 245.22 & 137.93 & 117.72 & 170.40 & 168.13 & \\
\hline dimensioning problem. & $J_{\text {total }}$ & 40.69 & 33.37 & 39.98 & 50.00 & 30.58 & 23.33 & 24.24 & 30.82 & EUR/100km \\
\hline
\end{tabular}

$J_{\text {total }}, J_{\text {fuel }}, J_{e l}$ and $J_{b a t}$ are the total optimization cost, fuel cost, cost for used electricity, and cost for battery, respectively;

$\delta$ is relative error in total cost; $c_{\text {rate }}$ is average charging rate from the chargers at the ends of the bus line.

The convex problem (15) minimizing the Complementary Hamiltonian will be referred to as $\mathrm{CH}$.

- Lower bound to the mixed-integer problem is obtained by solving the convex problem described in Section III-B. The results are given in the last row of Table I, but they are not further discussed in the paper.

\section{A. Problem setup}

The studied PHEV is equipped with a $220 \mathrm{~kW}$ EM and a $180 \mathrm{~kW}$ EGU as in Fig. 4. Its battery can be either energy optimized with cell capacity of $44 \mathrm{Ah}$ and cost of $500 \mathrm{EUR} / \mathrm{kWh}$, or power optimized with cell capacity of $10 \mathrm{Ah}$ and cost of $1500 \mathrm{EUR} / \mathrm{kWh}$. The allowed SOC range is within $25-75 \%$ and the operation is charge sustaining, where it is required to start and end at $50 \%$ SOC.

The battery sizing is investigated on four bus lines, from which the first one (L1) is given in Fig. 2, and the other three are certified emission test cycles available online ${ }^{1}$. The second bus line (L2) is the City Suburban Cycle, the third (L3) is the Orange County Bus Cycle and the forth (L4) is the Manhattan Bus Cycle repeated four times. On all four bus lines the vehicle can charge $4 \mathrm{~min}$ at each end and 2 min slide-in at about the middle. The chargers have maximum power of $100 \mathrm{~kW}$.

\footnotetext{
${ }^{1}$ The test cycles can be found on http://www.dieselnet.com/standards/cycles, March 2012.
}

\section{B. The global optimum}

The global optimum obtained by DP is given in the first row of Table I, while the cost vs. number of battery cells is shown in Fig. 6 and magnified around the optimum in Fig. 7. To make the comparison easier, the cost is given in [EUR/100km]. The results indicate that both the battery type and the bus line have significant impact on the optimal battery size. The power limit of the chargers, however, does not affect the battery size, as the vehicle never charges with rate greater than $63 \%$. This is because battery losses increase with charging power, and second, the batteries are too expensive to be large enough to accumulate the available grid energy. In the case of the $44 \mathrm{Ah}$ cells, it is the power limit of the cell that decides the battery size, since these cells never reach high state of charge, see Fig. 8(a). The battery with the 10 Ah cells is instead sized by its energy limit, see Fig. 8(b).

The dimensioning problem is solved by running DP at gridded values of the battery size, where the grid is made denser around the global optimum. Within the magnified regions shown in Fig. 7, there are in total 176 battery sizes for all four bus lines and the two battery types (see the dot marker). The high number of investigated battery sizes is needed to improve the solution accuracy, because near the optimum the cost may not vary much with battery size and a difference of 100 battery cells may give just $1 \%$ change in 


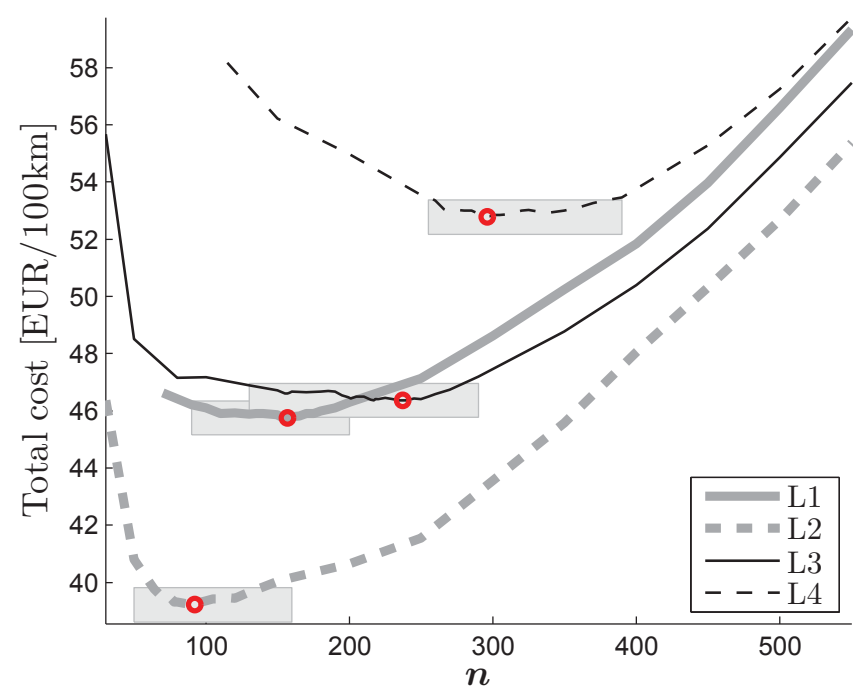

(a) Battery cell capacity is $44 \mathrm{Ah}$.

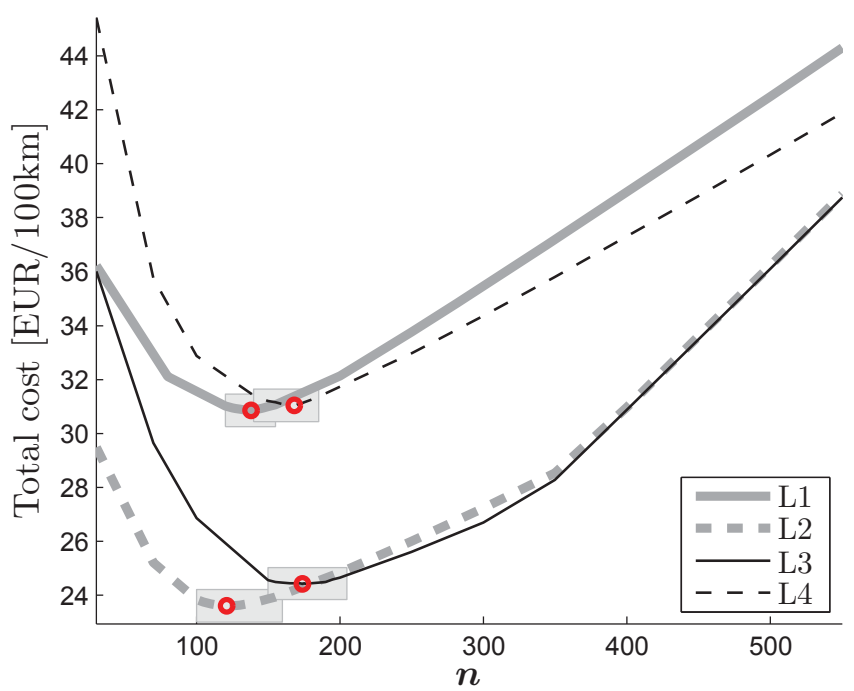

(b) Battery cell capacity is $10 \mathrm{Ah}$.

Fig. 6. Optimal cost vs. battery size obtained by Dynamic Programming. The circles indicate the global optimum of the battery dimensioning problem. The shaded regions around the optimum are magnified in Fig. 7.

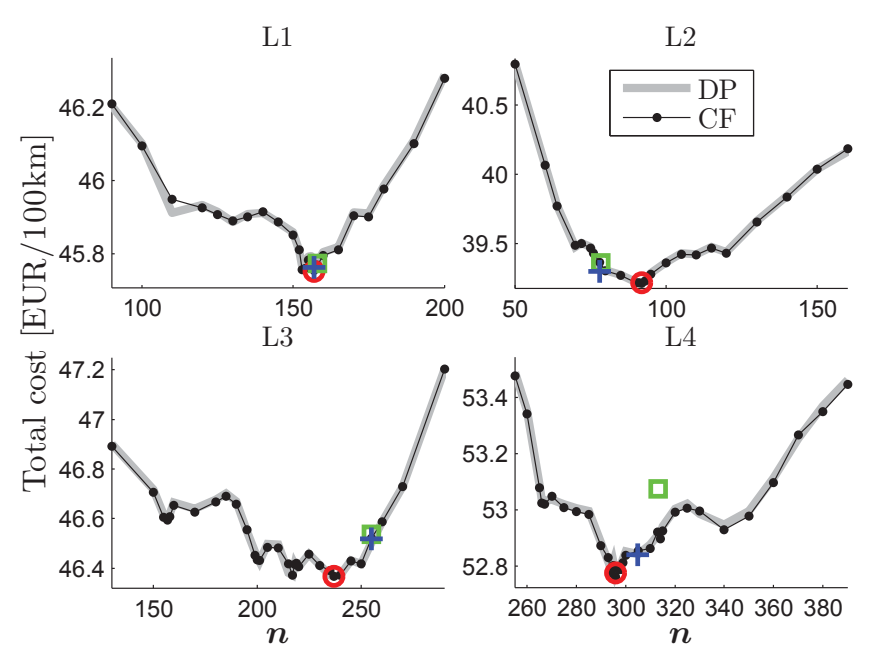

(a) Battery cell capacity is $44 \mathrm{Ah}$.

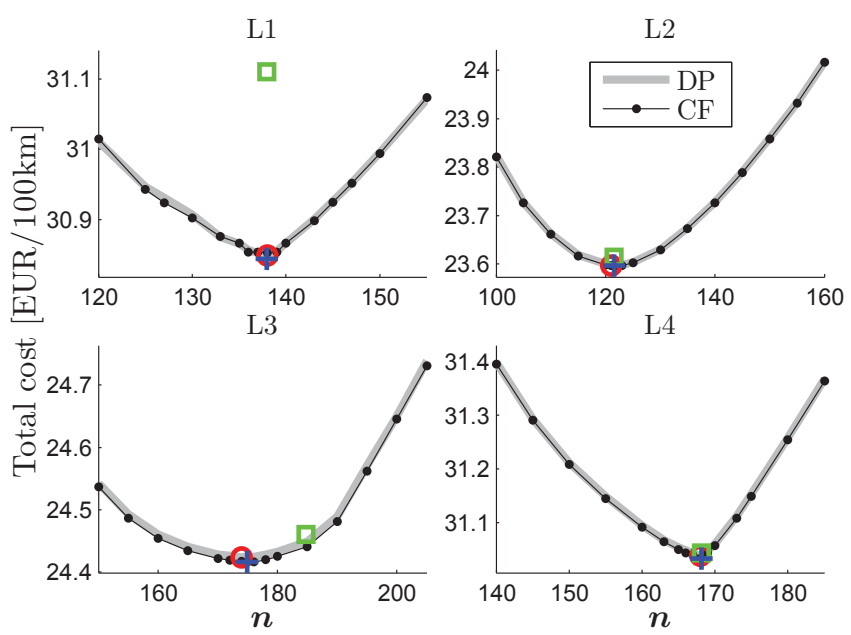

(b) Battery cell capacity is $10 \mathrm{Ah}$.

Fig. 7. Optimal cost vs. battery size. The solution of the mixed-integer control problem solved by DP for fixed battery sizes is indicated by the thick line, while the global optimum of the dimensioning problem is depicted by the circle. The optimal solution of the costate method (using CF) for the mixed-integer control problem is found at the fixed battery sizes indicated by the dot marker. The solution of the costate method (using CS) for simultaneous dimensioning and control is indicated by the plus. The initial solution of the latter costate method, where the initial engine on/off control is obtained from (10), is indicated by the square.

total cost (see Fig. 6). For each fixed battery size, the algorithm requires about $1.5-2.5 \mathrm{~h}$ on a standard $\mathrm{PC}(2.67 \mathrm{GHz}$ dual core CPU and 4 GB RAM), when configured for a highly accurate result. Hence, to keep the computation time within reasonable limits, the problem is solved using a computer cluster.

\section{Results from the costate method}

To investigate the performance of the costate method on the mixed-integer optimal control problem, $\mathrm{CF}$ is solved at the 176 fixed battery sizes that were also used in DP. Moreover, to better test the convergence of the costate method, the trivial initial solution is used that keeps the engine on at all time instances.
The results are illustrated in Fig. 7 and show that the global optimum of the mixed-integer control problem (for fixed battery sizes) is reached for all the 176 battery sizes. More specifically, it was found that the distance to the global optimum (represented as relative error in total cost)

$$
\delta=\frac{\text { cost }_{\text {costate_method }}-\text { cost }_{\mathrm{DP}}}{\text { cost }_{\mathrm{DP}}}
$$

is below $0.03 \%$ for all the investigated cases. For more than $90 \%$ of the cases the costate method gives even better result than DP (negative values in Table I). This is possibly due to the discretization error in DP, or the error due to variable scaling in CF. Detailed results for the optimal battery sizes obtained by the costate method are shown in the second row of Table 


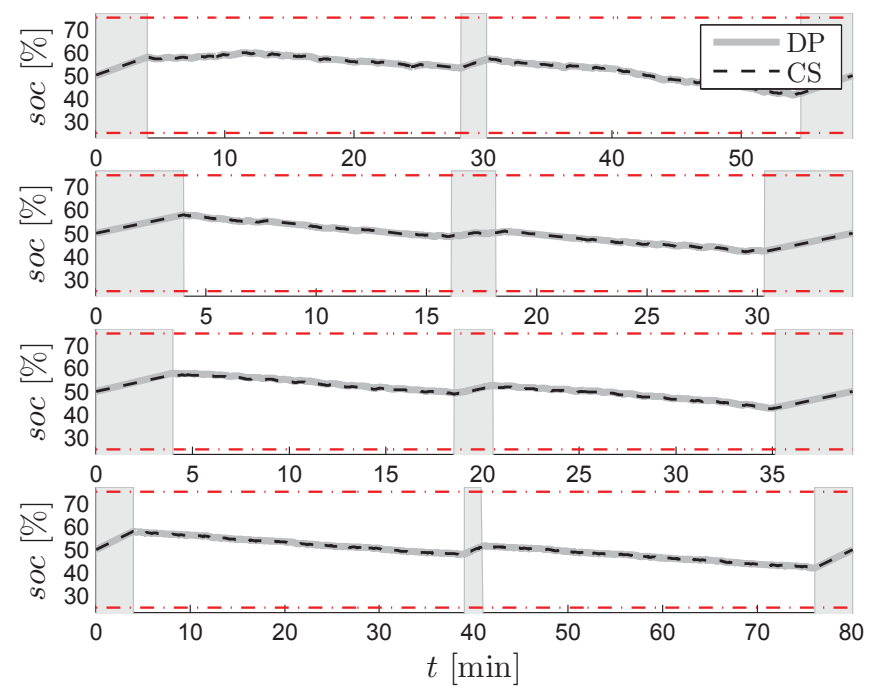

(a) Battery cell capacity is $44 \mathrm{Ah}$.

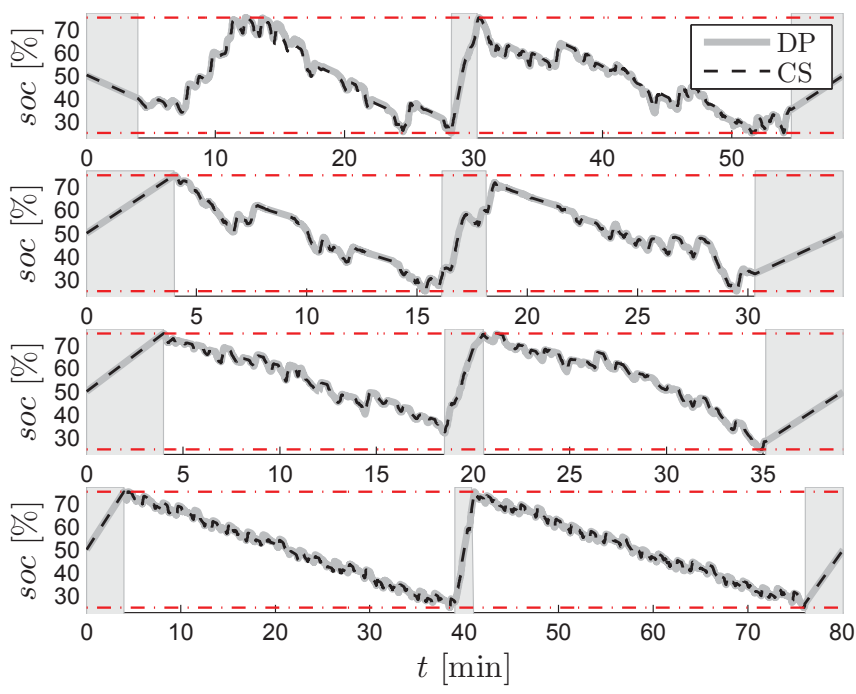

(b) Battery cell capacity is $10 \mathrm{Ah}$.

Fig. 8. Optimal battery SOC trajectories obtained by Dynamic Programming (DP) and convex optimization of the simultaneous sizing and control sub-problem (CS) using costate heuristics for the engine on/off control. The four plots, from top to bottom, represent the four bus lines L1 to L4. Charging opportunities are depicted by the shaded regions.

I.

One execution of $\mathrm{CF}$ requires about $4-10 \mathrm{~s}$, while one execution of $\mathrm{CH}$ needs about 4-10 s, depending on the bus line. The costate method requires in average 20 executions of $\mathrm{CF}$ and 10 executions of $\mathrm{CH}$. Hence, the optimal control for a fixed battery can be obtained in less than $5 \mathrm{~min}$.

\section{Simultaneous dimensioning and control}

In this section the costate method is used to solve the problem of simultaneous dimensioning and control when the battery size is a real-valued optimization variable. The purpose of this example is to show that the proposed costate method may not perform well on the control problem with design parameters.

First, an initial solution is chosen with the engine turned on at all time instances. The investigated PHEV is driven on L3 using the energy optimized battery cells, and the successive steps of the costate algorithm are illustrated in Fig. 9(a).

This example shows that the costate method is not able to move the battery size further from the initial value. There are two reasons for this; one is that at this stage of development no mechanism has been implemented to improve the battery size when minimizing the Complementary Hamiltonian, as discussed in Section IV-D. The other reason is a premature convergence that traps the solution in a local minimum. With the engine on at all time instances CS gives small battery size. Then, for this battery size the Complementary Hamiltonian suggests many $\left(N_{f}\right)$ time instances at which the engine on/off control flips value, thus drastically decreasing the total cost. In the next step when the Hamiltonian is minimized for the recently obtained on/off control, the solution is already trapped in a local minimum and CS gives a battery size that is close to the initial one. In certain cases the algorithm may still converge to the global optimum, as illustrated in the example in Fig. 9(b). However, a general conclusion can be drawn that the costate method may decrease the initial cost by improving the engine on/off control, but it may not be able to improve the design parameter, so it may be crucial to start with an initial battery size that is close to the optimal one.

Second, the costate method is evaluated with a better initial solution where the initial engine on/off control is obtained from (10). It can be noticed in the fourth row of Table I that this initial solution is already within $0.9 \%$ to the global optimum, thus supporting the same outcome observed in [15] that even this simple heuristic choice is a viable approach when dimensioning PHEVs with a series topology. Starting from this feasible solution, the costate method further decreases the difference in total cost to less than $0.35 \%$, which has been shown in the third row of Table I.

The optimal SOC trajectories of both DP and the costate method are shown in Fig. 8. It can be seen that both algorithms point to the same solution, as the lines almost completely overlap.

The total computation time needed to solve the problem of simultaneous dimensioning and control is less than $16.3 \mathrm{~min}$. Up to $10 \mathrm{~min}$ of this time are needed to obtain the initial solution. This is because 30 power levels are investigated to obtain the optimal power threshold above which the engine is turned on, and for each power level CS is solved in about $8-20 \mathrm{~s}$, depending on the bus line. The remaining $6.3 \mathrm{~min}$ are due to the costate method that requires executing $\mathrm{CS}$ and $\mathrm{CH}$, 15 and 8 times in average, respectively.

\section{DISCUSSION AND FUTURE WORK}

This section points out the key benefits of the proposed methodology and discusses possibilities for further studies. 


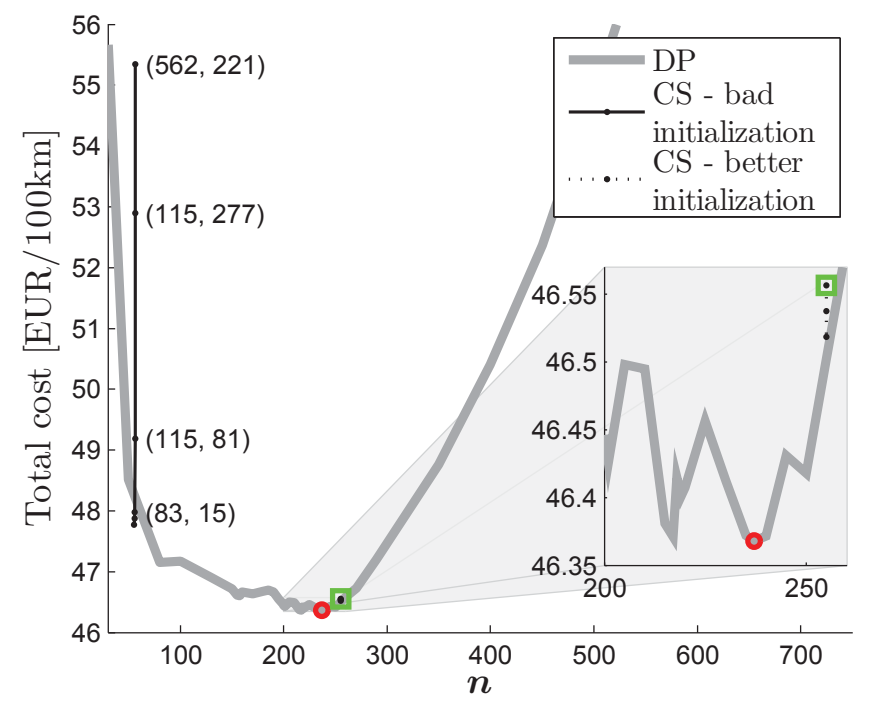

(a) Battery cell capacity is $44 \mathrm{Ah}$.

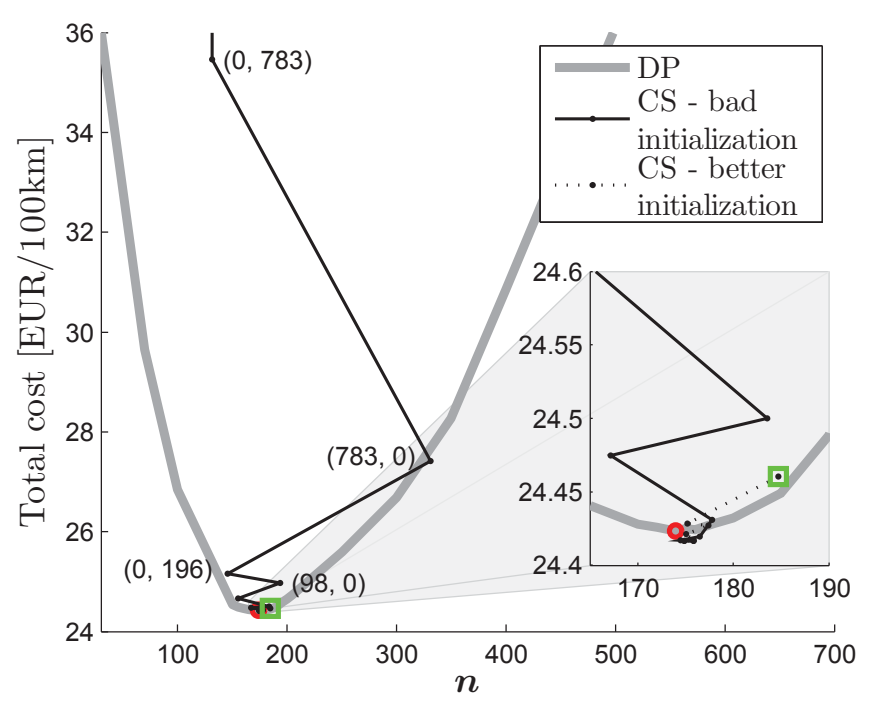

(b) Battery cell capacity is $10 \mathrm{Ah}$.

Fig. 9. Successive steps of the costate method for simultaneous dimensioning and control of the PHEV driven on L3. The direction of movement is towards decreasing total cost. The thin solid and dotted lines show evaluations of the algorithm with an initial solution $e(t)=1, \forall t \in\left[t_{0}, t_{f}\right]$, and with an initial solution obtained from (10), respectively. The latter (better) initial solution is indicated by a square. The boxed shaded regions to the right of the plots are magnifications around the global optimum indicated by a circle. The first and second number in parentheses show the number of time instances the engine is turned on and off, respectively, in the first few iterations of the algorithm.

\section{A. Multidimensional problems}

This study showed an alternative approach for solving the one-dimensional (one state) battery sizing and control problem. The simplicity of the problem allowed it to be solved with DP. In this study DP was configured to deliver very high accuracy that may not be necessary in industrial applications. Moreover, dedicated solvers exist [28] that may further decrease the computation time of DP to be comparable with the costate method.

However, the true advantage of the proposed method is in the possibility of optimizing multi-dimensional problems for which DP will need very long computation time. One example could be simultaneous dimensioning and control of a powertrain with two or more energy buffers, as investigated in [15], or an optimal control of a powertrain with thermal states of the vehicle components, such as the battery, EM, EGU, catalytic converter, passenger compartment. This is especially important for the electric components that could easily overheat if not managed properly. (An example illustrating convex modeling steps for including a thermal state in the model is given in [29].)

Each new state in the multidimensional model will introduce an additional costate in the Hamiltonian. This will affect the costate method and future work is needed to extend the method to such cases.

\section{B. Future studies}

The examples in this study showed that using costate heuristics for the engine on/off control, the solution of the dimensioning problem (using CS) is close to the global optimum, while the control problem (CF) for a fixed battery size practically reaches the global optimum. Although experimental, these results are promising and certainly show the need for further studies to investigate in which cases the algorithm will converge and in which it may not.

The computation time required by the costate method is short, about $5 \mathrm{~min}$ for a fixed battery size, but it could be decreased even further. For example, instead of using $\mathrm{CH}$ that requires $4-10 \mathrm{~s}, \tilde{\mathcal{H}}^{*}(\cdot)$ can be solved analytically in milliseconds. Similarly, $\mathcal{H}^{*}(\cdot)$ could also be solved analytically, if it is known that the state is not activating any constraints, as with the $44 \mathrm{Ah}$ cell. Then, the costate is constant, it needs to be computed only once, and it can be found either by convex optimization, or by root finding algorithm that gives a costate which preserves the charge sustain operation [30].

Future studies may focus on extending the strategy on problems with more integer control variables, e.g. parallel powertrains that have gear as an additional integer control signal. If the set of discrete values for the integer variables is small, then the costate strategy can be applied immediately by constructing new Complementary Hamiltonian for each new discrete value. If the discrete set is large, then instead of using Complementary Hamiltonians, improved values for the integer variable could be obtained using solvers for integer problems or with DP. These solvers will need relatively short computation time, because the costates can be used to eliminate the need for the continuous states in the problem (of course the same assumption will be used that the optimal costates will not change much between two consecutive iterations of the proposed algorithm).

Investigations are also needed for problems where states are integer variables. For example, the PHEV transportation problem could be easily transformed into an integer state problem, if the model penalizes (or prevents) frequent engine 
TABLE II

PARAMETER VALUES.

\begin{tabular}{ll} 
Vehicle frontal area & $A=7.54 \mathrm{~m}^{2}$ \\
Aerodynamic drag coefficient & $c_{d}=0.7$ \\
Rolling resistance coefficient & $c_{r}=0.007$ \\
Wheel radius & $r=0.509 \mathrm{~m}$ \\
Final gear & $\gamma=4.7$ \\
Vehicle mass without the battery & $m=14.5 \mathrm{t}$ \\
EM inertia & $I_{E M}=2.3 \mathrm{kgm}^{2}$ \\
Inertia of final gear and wheels & $I=41.8 \mathrm{kgm}^{2}$ \\
Charging stations efficiency & $\eta=92 \%$ \\
Power used by auxiliaries & $P_{a}=7 \mathrm{~kW}$ \\
Fuel price & $w_{f}=0.11 \mathrm{EUR} / \mathrm{kWh}$ \\
Electricity price & $w_{c}=0.1 \mathrm{EUR} / \mathrm{kWh}$ \\
Sampling time & $1 \mathrm{~s}$ \\
\hline
\end{tabular}

on/off switching. An example of such model can be found in [15].

\section{CONCLUSION}

This paper presented a method for optimal control of mixedinteger problems, which for given feasible values of the integer variables are convex in the rest of the variables. The method allows the problem to be solved in relatively short time using convex optimization techniques, while obtaining a solution near the global optimum.

The method has been applied on the problem of optimal battery dimensioning and control of a PHEV bus where the only integer variable is the engine on/off control. The results showed that the problem can be solved in less than $17 \mathrm{~min}$ with less than $0.35 \%$ difference from the global optimum. Moreover, the results showed that the global optimum is practically reached (difference of less than $0.03 \%$ ) for the optimal control problem of a PHEV with fixed battery.

Future studies may focus on adapting the method to problems with more integer control signals and design parameters.

\section{APPENDIX A}

\section{DATA FOR THE TRANSPORTATION PROBLEM}

Denoting by $v(t)$ and $\alpha(t)$ the velocity and slope of the bus line, the angular velocity and torque demanded on the shaft between the EM and the differential can be computed as

$$
\begin{aligned}
& \omega(t)=\frac{\gamma}{r} v(t) \\
& T_{v}(\boldsymbol{n}, t)=\frac{g r}{\gamma}\left(m+\boldsymbol{n} m_{b c}\right)\left(c_{r} \cos \alpha(t)+\sin \alpha(t)\right) \\
& \quad+\frac{\rho A c_{d} r^{3}}{2 \gamma^{3}} \omega^{2}(t)+\left(I_{E M}+\frac{I}{\gamma^{2}}+\left(m+\boldsymbol{n} m_{b c}\right) \frac{r^{2}}{\gamma^{2}}\right) \dot{\omega}(t)
\end{aligned}
$$

where $g$ is gravitational acceleration, $\rho$ is air density, and the rest of the parameters are described in Table II. The model neglects the inertial effects of the EGU.

Traction power to the wheels is delivered by a $220 \mathrm{~kW}$ EM as in Fig. 10 (losses of the power electronics are considered within the EM).

The $10 \mathrm{Ah}$ and $44 \mathrm{Ah}$ cells have mass of $600 \mathrm{~g}$ and $900 \mathrm{~g}$, resistance of $0.9 \mathrm{~m} \Omega$ and $2 \mathrm{~m} \Omega$, maximum charging current of $300 \mathrm{~A}$ and $50 \mathrm{~A}$ and maximum discharging current of $1000 \mathrm{~A}$ and $50 \mathrm{~A}$, respectively. The additional mass due to packaging

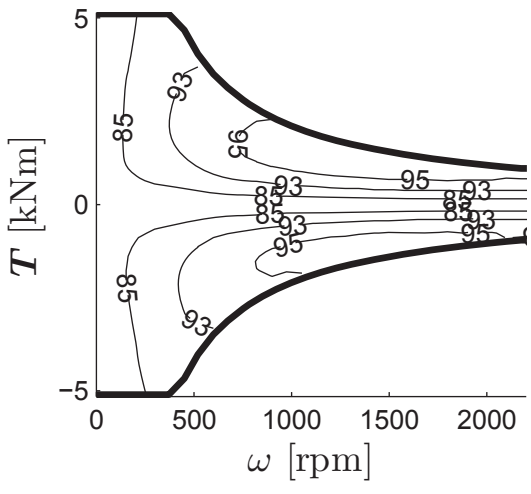

Fig. 10. Model of the EM. The thin lines represent efficiency and the thick lines are the torque bounds.

and circuitry is $14.5 \%$. The payment for both batteries is equally divided in $y=2$ years with $p=5 \%$ yearly interest rate. By denoting with $c_{b}$ the battery price in curency $/ \mathrm{kWh}$, the equivalent cell cost related to the driven bus line is obtained by multiplying the length of the bus line with the cell price per kilometer, given the average travel distance in one year $d=50000 \mathrm{~km}$. This yields

$$
w_{b}=c_{b} E_{s} m_{b c}\left(1+p \frac{y+1}{2}\right) \frac{\int_{t_{0}}^{t_{f}} v(t) d t}{y d}
$$

where $E_{s}[\mathrm{kWh} / \mathrm{kg}]$ is specific energy of the entire energy content of the battery cell.

\section{APPENDIX B}

\section{ANALYTICAL DERIVATION OF THE COSTATE}

To simplify the complex mathematical expressions below, the functions dependency on time will not be shown.

For a given battery size $n^{*}$ that minimizes $\mathcal{H}$, the torque of the EM can be uniquely determined via

$$
\boldsymbol{T}^{*}=\max \left\{\max \left\{T_{\min }, T_{b \min }\right\}, T_{v}\left(\boldsymbol{n}^{*}\right)\right\}
$$

where $T_{b \min }$ is the equivalent torque limit in the EM imposed by the battery charging with maximum current. It can be computed from (2) by considering zero EGU and grid power

$$
\begin{aligned}
T_{b m i n} \omega+P_{l o s s}\left(T_{b m i n}\right)+P_{a} & =P_{b m i n} \\
& =\left(V i_{c m i n}-R i_{c m i n}^{2}\right) \boldsymbol{n}^{*} .
\end{aligned}
$$

The EGU power can also be described from (2)

$$
\boldsymbol{P}_{\boldsymbol{g}}^{*}=\boldsymbol{T}^{*} \omega+P_{\text {loss }}\left(\boldsymbol{T}^{*}\right)+P_{a}-P_{b}\left(\boldsymbol{i}^{*}, \boldsymbol{n}^{*}\right)-\boldsymbol{P}_{\boldsymbol{c}}^{*}
$$

leaving only two variables, $\boldsymbol{i}^{*}$ and $\boldsymbol{P}_{c}^{*}$, at which the extremum of $\mathcal{H}$ will be investigated. If these variables are free, then at the extremum it holds

$$
\begin{aligned}
& \left(\frac{\partial \mathcal{H}}{\partial \boldsymbol{i}}\right)^{*}=-w_{f}\left(2 a_{o} \boldsymbol{P}_{\boldsymbol{g}}^{*}+a_{1}\right)\left(V-\frac{2 R}{\boldsymbol{n}^{*}} \boldsymbol{i}^{*}\right)-\boldsymbol{\lambda}^{*} V=0 \\
& \left(\frac{\partial \mathcal{H}}{\partial \boldsymbol{P}_{\boldsymbol{c}}}\right)^{*}=-w_{f}\left(2 a_{o} \boldsymbol{P}_{\boldsymbol{g}}^{*}+a_{1}\right)+\frac{w_{c}}{\eta}=0 .
\end{aligned}
$$

The costate can be computed at time instances covered by the following three cases: 
1) If the engine is on, there is a charging opportunity, and the constraints (7d), (7e) and (7f) are not active (whether a constraint is active, is decided with a tolerance of $0.01 \%$ of the expected signal's magnitude), the costate can be obtained by replacing $\boldsymbol{P}_{g}^{*}$ from (18) in (17), giving

$$
\lambda^{*}=-\frac{w_{c}}{\eta}\left(1-\frac{2 R}{\boldsymbol{n}^{*} V} \boldsymbol{i}^{*}\right) .
$$

2) If the engine is on, there is no charging opportunity and the constraints (7d) and (7e) are not active, then $\boldsymbol{P}_{\boldsymbol{c}}^{*}=0$ and the costate can be obtained from (17)

$$
\lambda^{*}=-w_{f}\left(2 a_{o} \boldsymbol{P}_{\boldsymbol{g}}^{*}+a_{1}\right)\left(1-\frac{2 R}{\boldsymbol{n}^{*} V} \boldsymbol{i}^{*}\right) .
$$

3) If the engine is off, there is a charging opportunity and the constraints (7d) and (7f) are not active, then

$$
\begin{gathered}
\boldsymbol{P}_{c}^{*}=\boldsymbol{T}^{*} \omega+P_{\text {loss }}\left(\boldsymbol{T}^{*}\right)+P_{a}-P_{b}\left(\boldsymbol{i}^{*}, \boldsymbol{n}^{*}\right) \\
\left(\frac{\partial \mathcal{H}}{\partial \boldsymbol{i}}\right)^{*}=-\frac{w_{c}}{\eta}\left(V-\frac{2 R}{\boldsymbol{n}^{*}} \boldsymbol{i}^{*}\right)-\boldsymbol{\lambda}^{*} V=0
\end{gathered}
$$

which gives expression for $\lambda^{*}$ exactly as in (19).

\section{APPENDIX C \\ DYNAMIC PROGRAMMING}

DP uses Bellman's principle of optimality [7] to solve the problem via backwards recursion handling nonlinearities and constraints in a straightforward way. The problem of battery sizing is solved by running DP at gridded values of the battery size. For each grid value $n_{j} \in \mathcal{N}$ the algorithm finds the optimal power split that minimizes the operational cost

$$
\begin{gathered}
J_{D P}^{*}\left(\boldsymbol{E}\left(t_{k}\right), t_{k}\right)=\min _{\boldsymbol{i}\left(t_{k}\right)}\left\{\int_{t_{k}}^{t_{k+1}} J(\boldsymbol{i}(t)) d t\right. \\
\left.\left.\quad+J_{D P}^{*}\left(\boldsymbol{E}\left(t_{k+1}\right), t_{k+1}\right)\right)\right\} \\
t_{k} \in \mathcal{T}, \quad \boldsymbol{i}\left(t_{k}\right) \in \mathcal{U}, \quad \boldsymbol{E}\left(t_{k}\right) \in \mathcal{X}, \quad t_{k} \in \mathcal{T}
\end{gathered}
$$

where $t_{k}$ and $t_{k+1}$ are consecutive time instances and $J_{D P}^{*}\left(\boldsymbol{E}\left(t_{k}\right), t_{k}\right)$ is a cost matrix holding the optimal cost-togo from state $\boldsymbol{E}\left(t_{k}\right)$ at time $t_{k}$ to the desired final state at time $t_{f}$. The sets $\mathcal{N}, \mathcal{T}, \mathcal{X}$ and $\mathcal{U}$ are discrete and the grid resolution determines the accuracy of the solution. Hence, to obtain an accurate solution, both the state and the current are gridded with 2000 points.

The operational cost $J(i(t))$ is computed as in (6), but with $\boldsymbol{i}(t)$ as a single control variable. Note that for a fixed battery size, the same procedure can be applied as in Appendix B to decrease the number of control variables. Moreover, the need for $\boldsymbol{P}_{\boldsymbol{c}}(t)$ as a control variable in (16) can also be eliminated, because the EGU will be turned on only if the vehicle cannot satisfy the driving demands by the electric grid alone.

The optimization is subject to the same constraints as in (7), except the constraint for ending at the desired SOC value (7i) which is formulated as a soft constraint

$$
J_{D P}^{*}\left(\boldsymbol{E}\left(t_{f}\right), t_{f}\right)=1000 \mid \boldsymbol{E}\left(t_{f}\right)-\text { soc }_{0} Q V \boldsymbol{n}_{\boldsymbol{j}} \mid .
$$

The infeasible points in $J_{D P}^{*}\left(\boldsymbol{E}\left(t_{k}\right), t_{k}\right)$ have been given a cost of 1000 and the sampling time is $1 \mathrm{~s}$.

\section{APPENDIX D}

\section{THE CONVEX SUB-PROBLEM (CS)}

The convex sub-problem (7), where $e(t)$ is not a decision variable, can be written in discrete time as

$$
\begin{aligned}
& \text { minimize } \\
& h \sum_{0}^{N-1} J\left(\boldsymbol{P}_{\boldsymbol{g}}(k), \boldsymbol{P}_{\boldsymbol{c}}(k), e(k)\right)+w_{b} \boldsymbol{n} \\
& \text { subject to, } \forall k \in\{0, \ldots, N-1\} \text {, } \\
& \boldsymbol{T}(k) \geq \max \left\{T_{\min }(\omega(k)), T_{v}(\boldsymbol{n}, k)\right\} \\
& \boldsymbol{T}(k) \omega(k)+P_{\text {loss }}(\boldsymbol{T}(k), k) \\
& \leq P_{b}(\boldsymbol{i}(k), \boldsymbol{n})+\boldsymbol{P}_{\boldsymbol{g}}(k) e(k)+\boldsymbol{P}_{\boldsymbol{c}}(k) c(k)-P_{a} \\
& \boldsymbol{i}(k) \in\left[i_{\text {cmin }}, i_{\text {cmax }}\right] \boldsymbol{n} \\
& \boldsymbol{P}_{\boldsymbol{g}}(k) \in\left[0, P_{\text {gmax }}\right] \\
& \boldsymbol{P}_{\boldsymbol{c}}(k) \in\left[0, \eta P_{\text {cmax }}\right] \\
& \boldsymbol{E}(k+1)=\boldsymbol{E}(k)-h V \boldsymbol{i}(k) \\
& \boldsymbol{E}(k) \in\left[\operatorname{soc}_{\min }, \operatorname{soc}_{\max }\right] Q V \boldsymbol{n} \\
& \boldsymbol{E}(N)=\boldsymbol{E}(0)=\operatorname{soc}_{0} Q V \boldsymbol{n} \\
& \boldsymbol{n}>0
\end{aligned}
$$

where $h$ is the sampling time and $N$ is the total number of samples.

The decision variables are scaled and a parser is used, CVX [31], to translate the problem into a form required by the solver SeDuMi [22].

\section{ACKNOWLEDGMENT}

The DP computations were performed in the Chalmers Center for Computational Science and Engineering (C3SE).

\section{REFERENCES}

[1] L. Guzzella and A. Sciarretta, Vehicle propulsion systems, introduction to modeling and optimization, 2nd ed. Berlin, Heidelberg: Springer, 2007.

[2] "AutoTram: Transport system of the future," Fraunhofer Institute for Transportation and Infrastructure Systems IVI, Tech. Rep., 2010.

[3] "On-line electric vehicle," Korea Advanced Institute of Science and Technology (KAIST), Tech. Rep., 2009.

[4] M. Johansson and O. Olsson, "Feasibility study of dual-mode buses in Gothenburg's public transport," Master's thesis, Chalmers University of Technology, Gothenburg, Sweden, 2011.

[5] L. Johannesson, S. Pettersson, and B. Egardt, "Predictive energy management of a 4QT series-parallel hybrid electric bus," Control Engineering Practice, vol. 17, no. 12, pp. 1440-1453, December 2009.

[6] T. C. Moore, "HEV control strategy: Implications of performance criteria, system configuration and design, and component selection," in Proceedings of the American Control Conference, Albuquerque, New Mexico, June 1997.

[7] R. Bellman, Dynamic Programming. New Jersey: Princeton Univ Pr, June 1957.

[8] U. Zoelch and D. Schroeder, "Dynamic optimization method for design and rating of the components of a hybrid vehicle," International Journal of Vehicle Design, vol. 19, no. 1, pp. 1-13, 1998.

[9] M. Kim and H. Peng, "Power management and design optimization of fuel cell/battery hybrid vehicles," Journal of Power Sources, vol. 165, no. 2, pp. 819-832, 2007.

[10] O. Sundström, L. Guzzella, and P. Soltic, "Torque-assist hybrid electric powertrain sizing: From optimal control towards a sizing law," IEEE Transactions on Control Systems Technology, vol. 18, no. 4, pp. 837849, July 2010. 
[11] M. Kim and H. Peng, "Combined control/plant optimization of fuel cell hybrid vehicles," in Proceedings of the 2006 American Control Conference Minneapolis, Minnesota, USA, June 14-16 2006.

[12] S. J. Moura, D. S. Callaway, H. K. Fathy, and J. L. Stein, "Tradeoffs between battery energy capacity and stochastic optimal power management in plug-in hybrid electric vehicles," Journal of Power Sources, vol. 195, no. 9, pp. 2979-2988, 2010.

[13] N. Murgovski, J. Sjöberg, and J. Fredriksson, "A methodology and a tool for evaluating hybrid electric powertrain configurations," Int. J. Electric and Hybrid Vehicles, vol. 3, no. 3, pp. 219-245, 2011.

[14] E. D. Tate and S. P. Boyd, "Finding ultimate limits of performance for hybrid electric vehicles," in SAE Technical Paper 2000-01-3099, 2000.

[15] N. Murgovski, L. Johannesson, J. Sjöberg, and B. Egardt, "Component sizing of a plug-in hybrid electric powertrain via convex optimization," Journal of Mechatronics, vol. 22, no. 1, pp. 106-120, 2012.

[16] S. Boyd and L. Vandenberghe, Convex Optimization. Cambridge University Press, 2004.

[17] L. S. Pontryagin, V. G. Boltyanskii, R. V. Gamkrelidze, and E. F. Mishchenko, The mathematical theory of optimal processes, L. W. Neustadt, Ed. Interscience publishers, 1962.

[18] W. R. Hamilton, "On a general method in dynamics," Philosophical Transactions of the Royal Society, vol. 2, pp. 247-308, 1834.

[19] — "Second essay on a general method in dynamics," Philosophical Transactions of the Royal Society, vol. 1, pp. 95-144, 1835.

[20] N. Murgovski, L. Johannesson, and J. Sjöberg, "Convex modeling of energy buffers in power control applications," in IFAC Workshop on Engine and Powertrain Control, Simulation and Modeling (E-CoSM), Rueil-Malmaison, Paris, France, October 23-25 2012.

[21] S. Sager, "Reformulations and algorithms for the optimization of switch- ing decisions in nonlinear optimal control," Journal of Process Control, vol. 19, no. 8, pp. 1238-1247, 2009.

[22] Y. Labit, D. Peaucelle, and D. Henrion, "SeDuMi interface 1.02: a tool for solving LMI problems with SeDuMi," IEEE International Symposium on Computer Aided Control System Design Proceedings, pp. 272-277, September 2002.

[23] G. A. Bliss, "The problem of Lagrange in the calculus of variations," American Journal of Mathematics, vol. 52, no. 4, pp. 673-744, 1930.

[24] E. J. McShane, "On multipliers for Lagrange problems," American Journal of Mathematics, vol. 61, no. 4, pp. 809-819, 1939.

[25] G. Paganelli, S. Delprat, T. M. Guerra, J. Rimaux, and J. Santin, "Control development for a hybrid-electric sport-utility vehicle: strategy, implementation and field test results," in Proceedings of the American Control Conference, 2001.

[26] - "Equivalent consumption minimization strategy for parallel hybrid powertrains," in IEEE Vehicular Technology Conference, 2002.

[27] A. E. Bryson and Y.-C. Ho, Applied Optimal Control. Taylor \& Francis Group, 1975.

[28] O. Sundström, D. Ambühl, and L. Guzzella, "On implementation of Dynamic Programming for optimal control problems with final state constraints," Oil Gas Sci. Technol., vol. 65, no. 1, pp. 91-102, 2009.

[29] N. Murgovski, L. Johannesson, A. Grauers, and J. Sjöberg, "Dimensioning and control of a thermally constrained double buffer plug-in HEV powertrain," in 51st IEEE Conference on Decision and Control, Maui, Hawaii, December 10-13 2012.

[30] S. Delprat, J. Lauber, T. M. Guerra, and J. Rimaux, "Control of a parallel hybrid powertrain: optimal control," IEEE Transactions on Vehicular Technology, vol. 53, no. 3, pp. 872-881, 2004.

[31] M. Grant and S. Boyd, "CVX: Matlab software for disciplined convex programming, version 1.21," http://cvxr.com/cvx, May 2010. 